\title{
Controlling Stored Products' Pests with Plant Secondary Metabolites: A Review
}

\author{
Polyxeni Nikolaou ${ }^{1}$, Paweł Marciniak ${ }^{1}\left(\mathbb{D}\right.$, Zbigniew Adamski $^{1,2}{ }^{(D)}$ and Nikoletta Ntalli ${ }^{3, *}$ \\ 1 Department of Animal Physiology and Development, Faculty of Biology, Adam Mickiewicz University, \\ Uniwersytetu Poznańskiego 6., 61-614 Poznań, Poland; polnik@amu.edu.pl (P.N.); \\ pmarcin@amu.edu.pl (P.M.); zbigniew.adamski@amu.edu.pl (Z.A.) \\ 2 Laboratory of Electron and Confocal Microscopy, Faculty of Biology, Adam Mickiewicz University, \\ Uniwersytetu Poznańskiego 6., 61-614 Poznań, Poland \\ 3 Department of Pesticides Control and Phytopharmacy, Benaki Phytopathological Institute, \\ 14561 Athens, Greece \\ * Correspondence: nntali@agro.auth.gr; Tel.: +30-6945901741
}

Citation: Nikolaou, P.; Marciniak, P.; Adamski, Z; Ntalli, N. Controlling Stored Products' Pests with Plant Secondary Metabolites: A Review. Agriculture 2021, 11, 879. https:// doi.org/10.3390/agriculture11090879

Academic Editor: Eduardo Rosa

Received: 25 July 2021

Accepted: 9 September 2021

Published: 14 September 2021

Publisher's Note: MDPI stays neutral with regard to jurisdictional claims in published maps and institutional affiliations.

Copyright: () 2021 by the authors. Licensee MDPI, Basel, Switzerland. This article is an open access article distributed under the terms and conditions of the Creative Commons Attribution (CC BY) license (https:// creativecommons.org/licenses/by/ $4.0 /)$.

\begin{abstract}
To date, only a handful of pesticides have been authorized by the European Council for the protection of stored grains. Resistance issues and ecotoxicity concerns necessitate the development of ecofriendly tools in that direction. In this review, we refer to the recent findings on plant extracts and pure plant-derived substances with promising biological activity and the potential to be used as biopesticides for stored products. The main aim of biopesticides is to be effective against target pests, without harming humans and the environment. Many plant species, among those reported herein, are part of the human diet, and are thus not harmful to humans. Edible plant extracts produced with inorganic solvents represent safe candidates for use as repellants, fumigants or contact pesticides. Cinnamon, rosemary, parsley, garlic, oregano and basil are found in products destined for human consumption but also display significant biological activities. Interestingly, cinnamon is one of the most widely tested botanical matrixes, exhibiting the best lethal effects on almost all insect and mite taxa reported herein (Acaroidea, Coleoptera and Lepidoptera), followed by basil and garlic. Prunus persica, Azadirachta indica A. Juss and Carum sp. seem to be very promising too as miticides and/or insecticides, with $A$. indica already being represented commercially by a plant-derived acaricidal formulation.
\end{abstract}

Keywords: biopesticides; plant extracts; stored grain pests; insects; mites; grains; commodities

\section{Introduction}

Over 800 million people worldwide suffer from malnutrition, and one-third of global food production is lost or wasted annually [1]. Pesticides are needed to control weeds, insect infestations and various pests and disease carriers (e.g., mosquitoes, ticks, rats and mice) in houses, offices, malls and streets [2]. Worldwide, approximately 2 million tons of pesticides are utilized annually [3]. However, the modes of action of pesticides are not always species-specific and exposure at even very low levels may have adverse health effects on humans. Additionally, concerns have been raised about environmental risks associated with exposure to these products through various routes (e.g., residues in food and drinking water) [2]. As a result, many pesticides have been withdrawn from the market. Interestingly, in an eight-year period (2001-2008), 26\% of insecticides were banned from the European Union due to unintentional impacts [4], extending as far as unbalancing/damaging an entire ecosystem [5].

Due to the constant increase of the population, larger quantities of cereals are required to cover the needs of the growing human population, thus making it important to find ways to minimize the loss of stored grains. Cereal grains are in fact the most basic ingredients of the human diet. According to the FAO, 'Cereals continue to be by far the most important source (in terms of calories) of total food consumption'. The food use of cereals has 
continued to increase, albeit at a decelerating rate. In developing countries, the per capita average is now $173 \mathrm{~kg}$, providing 56 percent of total calories [6]. On the other hand, insects feeding on stored grains can cause losses of up to 420 million tons annually [1]. There are two classes of arthropods-arachnids (Arachnida) and insects (Insecta) - infesting grains and food products. Within the first class, the subclass mites (Acari) contains some species of great economic importance. Among insects, species belonging to the orders of beetles (Coleoptera), moths and butterflies (Lepidoptera) are significant pests. Stored products may be damaged either through contamination by primary pests or through secondary contamination due to previously damaged grains; either caused by other pests (primary pests) or by being poorly threshed, dried and handled. Flour and milled rice might exhibit a reduction in weight and a decrease in quality due to an infestation of pests. Insects can encourage mold germination, increase the fatty acid percentage in the grain and can cause grain rancidity due to the uric acid they release, as well as causing grain pollution through their exuviae or feces. This, in consequence, can cause price discounts and shipping restrictions [1]. Traditionally, stored product insects have been controlled with synthetic insecticides, most of which are now out of the market due to ecotoxicological concerns (EC 1107/2006), as well as resistance issues [7,8]. To date, only a few of their active ingredients are still registered in the EU for stored product pest control. In particular, these include the fumigants magnesium phosphide and aluminum phosphide; the synthetic pyrethroids cypermethrine and deltamerthine, the phosphorothioate pirimiphos methyl and piperonyl butoxide applied by dusting [7]. Natural molecules of botanical origin have attracted international research interest in recent years as ecofriendly alternatives to their synthetic pesticidal ancestors (in commercial terms) [9]. Recent reviews on the use of the secondary metabolites of plants against stored product insects are those of Rajendran and co-workers, reporting the fumigant toxicity results conducted with essential oils of plants (mainly belonging to Apiaceae, Lamiaceae, Lauraceae and Myrtaceae) and their components (cyanohydrins, monoterpenoids, sulphur compounds, thiocyanates and others) [10], as well as that of Stejskal and co-workers, reporting on gas, liquid, gel and solid formulations of natural pesticides for stored-product applications [11].

In the present review, we report on the most important arthropod pests affecting grains and food products, along with the plant-derived substances reported to exhibit significant activity in the last 10 years. We categorized pests based on their taxonomic class and order, exhibiting similar habits, life cycles and metabolism. We aim to pinpoint the natural plant-derived substances that could be developed to combat stored-product pest infestations.

\section{Economically Important Groups of Stored-Product Pests and Plant-Derived Tools with Reported Activity}

\subsection{Mites (Acari)}

There are several mite species that cause severe losses to grains and stored products, mostly because they reproduce in large numbers, tolerate lower temperatures than insects and are not readily seen, causing great impact on storage facilities. Among the mites that are regarded as the most important pests of stored grains and food products are the species Gohieria fusca and Lepidoglyphus destructor (Glycyphagidae); Blomia freeman (Echimypodiae) and Chortoglyphus arcuatus (Chortoglyphidae), as well as Aleuroglyphus ovatus, Tyrophagus longior, Tyrophagus putrescentiae, Tyroborus lini and Acarus farris and Acarus siro of the Acaridae [12-15].

Traditionally, mites have been eradicated using synthetic miticides such as organophosphates, pyrethroids, pyridazines, juvenile hormone analogs and chitin synthesis inhibitors [13,16,17]. In addition, elevated $\mathrm{CO}_{2}$ concentrations are applied [18]. However, benzyl-benzoate, a substance that is produced and used industrially but which is also naturally produced by a range of plants - cinnamon and cassia (Cinnamomum spp.), carnation (Dianthus caryophyllus L.), hyacinths (Hyacinthus spp.), tuberose (Agave amica Medik.), common jasmine (Jasminum officinale L.) and Santos mahogany (Myroxylon balsamum L. Harms)-is often used as a commercial acaricide or as a reference substance in acaricidal tests. However, this substance is regarded as an allergen [19]. 
Azadirachtin (neem), a commercially available plant-derived pesticide obtained from the plant species Azadirachta indica A. Juss, is one of the most commonly used natural substances that causes lethal and sublethal insecticidal and miticidal effects. As reviewed by Collins (2006), it limits the growth of populations and causes mortality of T. putrescentiae, A. siro and L. destructor [13] but it proved to be less effective against five species of stored product mite pests than synthetic commercial miticides [20].

The components of clove bud (Eugenia caryophyllata Thumb.) oil-methyleugenol (median lethal dose $\left(\mathrm{LD}_{50}\right)=1.18 \mu \mathrm{g} / \mathrm{cm}^{2}$, isoeugenol, B-caryophyllene, eugenol and $\alpha$-humulene $\left(\mathrm{LD}_{50}=12.90 \mu \mathrm{g} / \mathrm{cm}^{2}\right)$ —showed lethal toxicity against $T$. putrescentiae, with methyleugenol and isoeugenol being more toxic than benzyl benzoate [21]. Furthermore, Rosmarinus officinalis L. essential oil (EO) and its constituent compounds were active against this mite, both as a fumigant $\left(\mathrm{LD}_{50}=8.24 \mu \mathrm{g} / \mathrm{cm}^{3}\right)$ and in contact toxicity $\left(\mathrm{LD}_{50}=5.49 \mu \mathrm{g} / \mathrm{cm}^{2}\right)$. The constituent camphor appeared to be successful in combating the pest (fumigant toxicity $\mathrm{LD}_{50}$ $=2.25 \mu \mathrm{g} / \mathrm{cm}^{3}$, contact toxicity $\mathrm{LD}_{50}=1.34 \mu \mathrm{g} / \mathrm{cm}^{2}$ ) more effectively than benzyl benzoate $\left(\mathrm{LD}_{50}=12.56 \mu \mathrm{g} / \mathrm{cm}^{3}\right.$, and $\left.9.03 \mu \mathrm{g} / \mathrm{cm}^{2}\right)$ [22]. Other constituent substances in rosemary oil are $\alpha$-pinene, 1,8-cineole and camphene, all exhibiting miticidal activity [23]. Lee (2015) proved the fumigant and contact toxicity of the essential oil of Ligustrum japonicum leaves against $T$. putrescentiae and calculated the respective $\mathrm{LD}_{50}$ values to be $16.48 \mu \mathrm{g} / \mathrm{cm}^{3}$ and $8.02 \mu \mathrm{g} / \mathrm{cm}^{2}$, respectively. $\alpha$-pinene is one of the compounds found in L. japonicum oil, showing the highest percentage [24]. These data indicate that camphor may be effective, as well as other compounds. However, camphor seems to be more toxic to mites than $\alpha$-pinene [21]. Ottoboni et al. (1992) reported that caraway (Carum carvi L.) essential oil was a significant candidate to combat $L$. destructor, G. fusca, A. siro and T. putrescentiae [25,26]. Furthermore 3,4-methylenedioxybenzene and its derivatives were described as successful miticides against, among others, T. putrescentiae [27]. Interestingly, apiol, which naturally occurs in the seeds of parsley (Petroselinum sativum Hoffm), was not toxic against T. putrescentiae, although it has been proven to be active against Dermatophagoides species. Essential oils obtained from both the aerial parts and seeds of the forget-me-not plant (Myosotis arvensis (L.)) or ingredient compounds used individually, namely 2,4,5-trimethylbenzaldehyde, 2,4-methylbenzaldehyde, 2,5-dimethylbenzaldehyde, 2methylbenzaldehyde, 2,3-dimethylbenzaldehyde, 3-methylbenzaldehyde, 4-methylbenzaldehyde, 3 -octanone, butyl isothiocyanate and nonanal, showed significantly greater contact and vapor toxicity against $T$. putrescentiae than benzyl benzoate [28]. T. putrescentiae was proven to be susceptible to the essential oil of garlic (Allium satioum L.), basil (Ocimum basilicum L.) and fenugreek (Trigonella foenum-graecum L.), in descending order, when exposed for one to three days [29]. However, the sulfide-rich garlic essential oil was toxic to Cheyletus malaccensis, a predatory mite and natural enemy of A. siro, T. putrescentiae and L. destructor [30]. Nonetheless, garlic essential oil and its active compounds can be used as possible miticides against a range of mites [31]. Unfortunately, some botanical extracts have been proven to be lethal for beneficial insects, such as citronella, eucalyptus, garlic, pyrethrum and neem. Sometimes their effects may be non-lethal, such as inhibiting natural enemies from utilizing prey, reducing prey availability, decreasing reproduction, inhibiting the ability of natural enemies to recognize prey, influencing the sex ratio (females:males) and reducing mobility. Nonetheless, detailed knowledge of the lethal or nonlethal effects of botanical pesticides on beneficial insects is essential for the sustainable control of insect pests and pollination activities for improved and sustainable agricultural production [32].

De Assis and co-authors (2011) tested the fumigant toxicity of eugenol and essential oils from cinnamon (Cinnamomum zeylanicum Blume), Surinam cherry (Eugenia uniflora L.), uvalha (Eugenia uvalha Cambess.), weeping paperbark (Melaleuca leucadendra (L.)), cake bush (Piper marginatum Jacq.) and Brazilian peppertree (Schinus terebinthifolia G.Raddi) against $T$. putrescentiae. The lowest median lethal concentration $\left(\mathrm{LC}_{50}\right)$ values were obtained for eugenol and $C$. zeylanicum essential oil. These data are in agreement, since eugenol is the major component of C. zeylanicum essential oil and its content may exceed $\frac{3}{4}$ of all the volatile ingredients of this oil [33]. The next most abundant component-linalool-is present in about nine times lower amounts [34]. Although it is not as important, linalool may increase toxic effects since it appeared to be toxic against $T$. longior in contact and 
fumigant toxicity studies. Similarly the toxicity of menthol, menthone, fenchone, linalyl acetate and eucalyptol, the most abundant substances in the essential oils of lavender (Lavandula angustifolia Mill, Lavandula stoechas L.), peppermint (Mentha x piperita L.) and eucalyptus (Eucaliptus globulus), should be assessed for their lethality [35].

The observation of the variable susceptibility of various species of mites to natural substances is also seen in the case of natural aldehydes used as miticides. This phenomenon was described for three natural aldehydes, namely, (2E)-hexenal, (2E, 6Z)-nonadienal and (2E)-nonenal, produced in plants from organic acids, when tested in feeding tests from 36 to $314 \mathrm{mg} / \mathrm{g}$ against $A$. siro, A. ovatus and T. putrescentiae. Specifically, the susceptibility of A. siro was similar for all three aldehydes, whereas T. putrescentiae was about eight times more susceptible to nonadienal than to hexenal, and was not significantly affected by nonenal [36]. In another study, benzaldehyde $\left(\mathrm{LD}_{50}=4.23 \mu \mathrm{g} / \mathrm{cm}^{2}\right)$ isolated from the peach Prunus persica, as well as salicylaldehyde $\left(\mathrm{LD}_{50}=1.02 \mu \mathrm{g} / \mathrm{cm}^{2}\right)$, cinnamaldehyde $\left(\mathrm{LD}_{50}=1.66 \mu \mathrm{g} / \mathrm{cm}^{2}\right)$ and phthaldialdehyde $\left(\mathrm{LD}_{50}=5.16 \mu \mathrm{g} / \mathrm{cm}^{2}\right)$, were tested against $T$. putrescentiae. All aldehydes exhibited better efficacies than benzyl benzoate $\left(\mathrm{LD}_{50}=9.75\right.$ $\left.\mu \mathrm{g} / \mathrm{cm}^{2}\right)$. However, the values calculated for P. persica essential oil $\left(\operatorname{LD}_{50}=11.23 \mu \mathrm{g} / \mathrm{cm}^{2}\right)$ were higher than those for benzyl benzoate [37]. This research shows that aldehydes and essential oils can be applied in grain and food protection. However, their application must be carefully adjusted to the tested species.

There are suggestions that jasmonic acid (JA) can be used as a miticide against mitepests of grains and stored food. This compound may affect mite reproduction and limit losses. The tomato mutants that were unable to accumulate JA were characterized by a higher rate of egg-hatching of mite-pests compared to the wild type. Therefore, JA was suggested as a substance of ovicidal activity [38]. Most interestingly, JA additionally attracts predatory mites and therefore it may decrease the level of pests [39]. These data seem to open a field of interesting further research on the species of interest.

Angiosperms also deliver bioactive substances that may be useful in limiting losses caused by mites. For instance, essential oils obtained from gymnosperm plants such as Pinus pinea, Pinus halepensis, Pinus pinaster and Pinus nigra were described as toxic for $T$. putrescentiae [40], with P. pinea being the most effective. Moreover, 1,8-cineole and limonene showed miticidal activity when tested at 8 or $6 \mu \mathrm{L}$ on $6 \mathrm{~cm}$ of filter paper. Likewise, Juniperus chinensis essential oil and its respective components were applied in impregnated disc biotests against $T$. putrescentiae, with the $\mathrm{LD}_{50}$ values calculated at 38.1, 15.33, and $42.85 \mu \mathrm{g} / \mathrm{cm}^{2}$ for the essential oil, bornyl acetate and $\alpha$-eudesmol, respectively [41]. Based on the effect and the content of the substances in the oil, the authors suggested that bornyl acetate is the major substance responsible for this acaricidal activity. The authors also reported that sabinene and $\alpha$-thujene were not toxic to the mites.

The abovementioned results prove that plant-derived extracts and single compounds may become interesting alternatives to commercial miticides. In many cases, they are obtained from plants that are nontoxic to humans, since they are part of the human diet, for example, garlic or parsley (Table 1). Therefore, they can be used in food stores, being relatively safe to humans, of course depending on the concentration used. 
Table 1. Plant species reported to exhibit significant activity against stored-product pests in recent years.

\begin{tabular}{|c|c|c|c|}
\hline \multicolumn{4}{|c|}{ Mites } \\
\hline Plant Species & Formulation & Pest Species & Reference \\
\hline \multirow{3}{*}{ Allium sativum } & essential oil & Tyrophagus putrescentiae & {$[29,31]$} \\
\hline & essential oil & Cheyletus malaccensis & {$[30]$} \\
\hline & Commercial product of neem & Tyrophagus putrescentiae & \\
\hline Azadirachta indica & (Fortune AZA) & $\begin{array}{c}\text { Acarus siro } \\
\text { anidoolonhus destructor }\end{array}$ & {$[13,20]$} \\
\hline Carum carvi & essential oil & $\begin{array}{c}\text { Lepidoglyphus destructor } \\
\text { Gohieria fusca } \\
\text { Lepidoglyphus destructor } \\
\text { Acarus siro } \\
\text { Tyrophagus putrescentiae }\end{array}$ & {$[25]$} \\
\hline Cinnamomum zeylanicum & essential oil & Tyrophagus putrescentiae & [33] \\
\hline Eucaliptus globulus & essential oil & Tyrophagus longior & [35] \\
\hline Eugenia caryophyllata & essential oil & Tyrophagus putrescentiae & [21] \\
\hline Eugenia uniflora & essential oil & Tyrophagus putrescentiae & [33] \\
\hline Eugenia wvalha & essential oil & Tyrophagus putrescentiae & [33] \\
\hline Juniperus chinensis & essential oil & Tyrophagus putrescentiae & [41] \\
\hline Lavandula angustifolia & essential oil & Tyrophagus longior & {$[35]$} \\
\hline Lavandula stoechas & essential oil & Tyrophagus longior & [35] \\
\hline Ligustrum japonicum & essential oil & Tyrophagus putrescentiae & {$[24]$} \\
\hline Melaleuca leucadendra & essential oil & Tyrophagus putrescentiae & [33] \\
\hline Mentha piperita & essential oil & Tyrophagus longior & [35] \\
\hline Myosotis arvensis & essential oil & Tyrophagus putrescentiae & [28] \\
\hline Ocimum basilicum & essential oil & Tyrophagus putrescentiae & [29] \\
\hline Petroselinum sativum & active constituents & Tyrophagus putrescentiae & [27] \\
\hline Pinus pinea & essential oil & Tyrophagus putrescentiae & [40] \\
\hline Pinus halepensis & essential oil & Tyrophagus putrescentiae & [40] \\
\hline Pinus pinaster & essential oil & Tyrophagus putrescentiae & [40] \\
\hline Pinus nigra & essential oil & Tyrophagus putrescentiae & [40] \\
\hline Piper marginatum & essential oil & Tyrophagus putrescentiae & [33] \\
\hline Prunus persica & essential oil & Tyrophagus putrescentiae & [37] \\
\hline Rosmarinus officinalis & essential oil & Tyrophagus putrescentiae & [22] \\
\hline Schinus terebinthifolius & essential oil & Tyrophagus putrescentiae & [33] \\
\hline Trigonella foenum-graecum & essential oil & Tyrophagus putrescentiae & [29] \\
\hline \multicolumn{4}{|c|}{ Coleoptera } \\
\hline Plant Species & Formulation & Pest Species & Reference \\
\hline Achillea wilhelmsii & essential oil & Tribolium castaneum & {$[42]$} \\
\hline Achyranthus aspera & essential oil & Cryptolestes ferrugineus & [43] \\
\hline Acisanthera. & ethanolic extract & Tenebrio molitor & [44] \\
\hline \multirow{2}{*}{ Acorus calamus } & \multirow{2}{*}{ essential oil } & Sitophilus oryzae & \multirow{2}{*}[45]{} \\
\hline & & Tribolium castaneum & \\
\hline Adenocalymma nodosum & ethanolic extract & Tenebrio molitor & [44] \\
\hline Agastache rugosa & plant extract & Tribolium castaneum & {$[46]$} \\
\hline \multirow{4}{*}{ Allium sativum } & powder & Oryzaephilus surinamensis & [47] \\
\hline & essential oil & Tenebrio molitor & [48] \\
\hline & \multirow{2}{*}{ essential oil } & Sitophilus oryzae & \multirow{2}{*}[49]{} \\
\hline & & Tribolium castaneum & \\
\hline Alpinia blepharocalyx & essential oil & Lasioderma serricorne & {$[50]$} \\
\hline Amoтum maximum & essential oil & Tribolium castaneum & [51] \\
\hline \multirow{2}{*}{ Amomum tsaoko } & \multirow{2}{*}{ essential oil } & Lasioderma serricorne & \multirow{2}{*}[52]{} \\
\hline & & Tribolium castaneum & \\
\hline
\end{tabular}


Table 1. Cont.

\begin{tabular}{|c|c|c|c|}
\hline \multicolumn{4}{|c|}{ Coleoptera } \\
\hline Plant Species & Formulation & Pest Species & Reference \\
\hline Anethum graveolens & essential oil & Sitophilus zeamais & [53] \\
\hline Armoracia rusticana & essential oil & Sitophilus zeamais & [54] \\
\hline Artemisia absinthium & powder & Oryzaephilus surinamensis & [47] \\
\hline \multirow{2}{*}{ Artemisia anethoides } & \multirow{2}{*}{ essential oil } & Lasioderma serricorne & \multirow{2}{*}{ [55] } \\
\hline & & Tribolium castaneum & \\
\hline \multirow{2}{*}{ Artemisia herba-alba } & \multirow{2}{*}{ essential oil } & Oryzaephilus surinamensis & \multirow{2}{*}{ [56] } \\
\hline & & Tribolium castaneum & \\
\hline Artemisia judaica & \multirow{2}{*}{ essential oil } & \multirow{2}{*}{ Sitophilus oryzae } & \multirow{2}{*}{ [57] } \\
\hline Artemisia monosperma & & & \\
\hline Artemisia vulgaris & essential oil & Sitophilus zeamais & [58] \\
\hline \multirow{2}{*}{ Artemisia stolonifera } & \multirow{2}{*}{ essential oil } & Lasioderma serricorne & \multirow{2}{*}{ [59] } \\
\hline & & Tribolium castaneum & \\
\hline \multirow{2}{*}{ Aster ageratoides } & \multirow{2}{*}{ essential oil } & Sitophilus zeamais & \multirow{2}{*}{ [60] } \\
\hline & & Tribolium confusum & \\
\hline Astoma seselifolium & essential oil & Sitophilus oryzae & {$[57]$} \\
\hline \multirow{2}{*}{ Atalantia guillauminii } & \multirow{2}{*}{ essential oil } & Lasioderma serricorne & \multirow{2}{*}[61]{} \\
\hline & & Tribolium castaneum & \\
\hline \multirow{2}{*}{ Azadirachta indica } & essential oil & \multirow{2}{*}{ Tribolium castaneum } & \multirow{2}{*}[62]{} \\
\hline & seed oil & & \\
\hline Bauhinia purpurea & methanol extract & Trogoderma granarium & [63] \\
\hline Bidens sulphurea & ethanolic extract & Tenebrio molitor & {$[44]$} \\
\hline Caesalpinia gilliesii & methanol extract & Trogoderma granarium & [63] \\
\hline \multirow{3}{*}{ Carum carvi } & \multirow{3}{*}{ essential oil } & Tribolium castaneum & \multirow{3}{*}[64]{} \\
\hline & & Sitophilus oryzae & \\
\hline & & Rhizopertha dominica & \\
\hline \multirow{2}{*}{ Carum copticum } & essential oil & Sitophilus granarius & [65] \\
\hline & & Tribolium confusum & \\
\hline Caryopteris incana & essential oil & Sitophilus zeamais & [66] \\
\hline Calendula officinalis & essential oil & Sitophilus granarius & [67] \\
\hline Callistemon viminals & essential oil & Sitophilus oryzae & {$[57]$} \\
\hline Capsicum annuum & plant extract & Tribolium castaneum & {$[46]$} \\
\hline Cassia fistula & methanol extract & Trogoderma granarium & [63] \\
\hline & water extract & & \\
\hline Cassia occidentalis & ethanol extract & Oryzaephilus surinamensis & [68] \\
\hline & acetone extract & & \\
\hline Cassia senna & methanol extract & Trogoderma granarium & [63] \\
\hline Cayratia japonica & essential oil & Sitophilus zeamais & [69] \\
\hline cayrusu juротыса & & Tribolium castaneum & \\
\hline Chenopodium album & ether extract & Oryzaephilus surinamensis & {$[70]$} \\
\hline Chrysanthemum frutescens & methanol extract & Trogoderma granarium & [63] \\
\hline
\end{tabular}


Table 1. Cont.

\begin{tabular}{|c|c|c|c|}
\hline \multicolumn{4}{|c|}{ Coleoptera } \\
\hline Plant Species & Formulation & Pest Species & Reference \\
\hline \multirow{4}{*}{ Cinnamomum verum } & \multirow{4}{*}{ essential oil } & Tenebrio molitor & [71] \\
\hline & & Tribolium castaneum & \multirow{3}{*}[64]{} \\
\hline & & Sitophilus oryzae & \\
\hline & & Rhizopertha dominica & \\
\hline Citrus aurantifolia & essential oil & Sitophilus oryzae & [57] \\
\hline Citrus lemon & essential oil & Sitophilus oryzae & [57] \\
\hline Citrus medica & essential oil & Tribolium castaneum & [72] \\
\hline Citrus paradisi & essential oil & Rhizopertha dominica & [73] \\
\hline \multirow{6}{*}{ Citrus reticulata } & essential oil & Tribolium confusum & [74] \\
\hline & powder & \multirow{3}{*}{ Tribolium castaneum } & \multirow{2}{*}[46,75]{} \\
\hline & ethanol extract & & \\
\hline & essential oil & & [76] \\
\hline & essential oil & Cryptolestes ferrugineus & {$[77]$} \\
\hline & essential oil & Rhyzopertha dominica & [73] \\
\hline \multirow{4}{*}{ Citrus sinensis } & essential oil & Tribolium castaneum & {$[76]$} \\
\hline & essential oil & Rhizopertha dominica & [78] \\
\hline & essential oil & Sitophilus oryzae & [57] \\
\hline & essential oil & Sitophilus zeamais & {$[79]$} \\
\hline Calamintha glandulosa & essential oil & Tribolium castaneum & [80] \\
\hline Clausena anisum-olens & essential oil & Lasioderma serricorne & [81] \\
\hline \multirow{3}{*}{ Cleome viscosa } & water extract & \multirow{3}{*}{ Oryzaephilus surinamensis } & \multirow{3}{*}[68]{} \\
\hline & ethanol extract & & \\
\hline & acetone extract & & \\
\hline Coriandrum sativum & essential oil & Sitophilus oryzae & [82] \\
\hline \multirow{5}{*}{ Crithmum maritimum } & \multirow{5}{*}{ essential oil } & Oryzaephilus surinamensis & \multirow{5}{*}{ [83] } \\
\hline & & Sitophilus granarius & \\
\hline & & Sitophilus oryzae & \\
\hline & & Tribolium castaneum & \\
\hline & & Tribolium confusum & \\
\hline Cuminum сутіпит & essential oil & Sitophilus zeamais & [53] \\
\hline \multirow{2}{*}{ Cupressus lusitanica } & \multirow{2}{*}{ essential oil } & Sitophilus zeamais & \multirow{2}{*}{ [84] } \\
\hline & & Tribolium castaneum & \\
\hline Cupressus macrocarpa & essential oil & Sitophilus oryzae & [57] \\
\hline Cupressus sempervirens & essential oil & Sitophilus oryzae & {$[57]$} \\
\hline \multirow{2}{*}{ Cymbopogon citratus } & essential oil & Sitophilus oryzae & [85] \\
\hline & essential oil & Tribolium castaneum & [86] \\
\hline Cymbopogon giganteus & essential oil & Tribolium castaneum & {$[86]$} \\
\hline Cymbopogon schoenanthus & essential oil & Tribolium castaneum & {$[86]$} \\
\hline \multirow{3}{*}{ Cymbopogon winterianus } & \multirow{3}{*}{ essential oil } & Tribolium castaneum & \multirow{3}{*}[64]{} \\
\hline & & Sitophilus oryzae & \\
\hline & & Rhizopertha dominica & \\
\hline
\end{tabular}


Table 1. Cont.

\begin{tabular}{|c|c|c|c|}
\hline \multicolumn{4}{|c|}{ Coleoptera } \\
\hline Plant Species & Formulation & Pest Species & Reference \\
\hline \multirow{2}{*}{ Dahlia pinnata } & \multirow{2}{*}{ essential oil } & Sitophilus oryzae & \multirow{2}{*}[50]{} \\
\hline & & Sitophilus zeamais & \\
\hline Dennettia tripetala & essential oil & Sitophilus oryzae & [87] \\
\hline Dimorphandra mollis & ethanolic extract & Tenebrio molitor & {$[44]$} \\
\hline \multirow{2}{*}{ Dracocephalum moldavica } & \multirow{2}{*}{ essential oil } & Sitophilus zeamais & \multirow{2}{*}{ [88] } \\
\hline & & Tribolium confusum & \\
\hline Drimys winteri & essential oil & Tribolium castaneum & [89] \\
\hline Dictamnus dasycarpus & essential oil & Lasioderma serricorne & [90] \\
\hline Eruca sativa & essential oil & Tribolium confusum & {$[74]$} \\
\hline Etlingera yunnanensis & essential oil & Tribolium castaneum & {$[91]$} \\
\hline \multirow{2}{*}{ Eucalyptus camaldulensis } & \multirow{2}{*}{ essential oil } & Sitophilus oryzae & \multirow{2}{*}[92,93]{} \\
\hline & & Tribolium castaneum & \\
\hline Eucalyptus citriodora & essential oil & Tribolium castaneum & [86] \\
\hline \multirow{2}{*}{ Eucalyptus floribundi } & \multirow{2}{*}{ essential oil } & Oryzaephilus surinamensis & \multirow{2}{*}{ [94] } \\
\hline & & Rhizopertha dominica & \\
\hline \multirow{3}{*}{ Eucalyptus globulus } & essential oil & Tribolium confusum & [74] \\
\hline & \multirow{2}{*}{ essential oil } & Lasioderma serricorne & \multirow{2}{*}[95]{} \\
\hline & & Rhizopertha dominica & \\
\hline \multirow{2}{*}{ Eucalyptus intertexta } & \multirow{2}{*}{ essential oil } & Sitophilus oryzae & \multirow{2}{*}{ [93] } \\
\hline & & Tribolium castaneum & \\
\hline \multirow{2}{*}{ Eucalyptus leucoxylon } & \multirow{2}{*}{ essential oil } & Sitophilus oryzae & \multirow{2}{*}[96]{} \\
\hline & & Tribolium castaneum & \\
\hline Eucalyptus obliqua & essential oil & Sitophilus oryzae & {$[82]$} \\
\hline Eucalyptus procera & essential oil & Tribolium castaneum & [97] \\
\hline \multirow{2}{*}{ Eucalyptus saligna } & \multirow{2}{*}{ essential oil } & Sitophilus zeamais & \multirow{2}{*}{ [84] } \\
\hline & & Tribolium castaneum & \\
\hline \multirow{2}{*}{ Eucalyptus sargentii } & \multirow{2}{*}{ essential oil } & Sitophilus oryzae & [93] \\
\hline & & Tribolium castaneum & \\
\hline Euonymus japonicus & methanol extract & Trogoderma granarium & [63] \\
\hline Ferula narthex & essential oil & Cryptolestes ferrugineus & [43] \\
\hline & essential oil & Sitophilus zeamais & {$[53]$} \\
\hline Foeniculum vulgare & & Tribolium castaneum & \\
\hline & essential oil & Sitophilus oryzae & [64] \\
\hline & & Rhizopertha dominica & \\
\hline Ginkgo biloba & plant extract & Tribolium castaneum & [46] \\
\hline & & Rhizopertha dominica & \\
\hline Hyptis suaveolens & essential oil & Sitophilus oryzae & [98] \\
\hline & & Tribolium castaneum & \\
\hline Juniperus formosana & essential oil & Tribolium castaneum & [99] \\
\hline
\end{tabular}


Table 1. Cont.

\begin{tabular}{|c|c|c|c|}
\hline \multicolumn{4}{|c|}{ Coleoptera } \\
\hline Plant Species & Formulation & Pest Species & Reference \\
\hline Juniperus polycarpus & essential oil & Tribolium confusum & \multirow{2}{*}{ [100] } \\
\hline Juniperus sabina & essential oil & Tribolium confusum & \\
\hline Kadsura heteroclita & essential oil & Sitophilus zeamais & [101] \\
\hline \multirow{2}{*}{ Laurelia sempervirens } & essential oil & Sitophilus zeamais & [102] \\
\hline & essential oil & Tribolium castaneum & [89] \\
\hline \multirow{3}{*}{ Laurus nobilis } & \multirow{3}{*}{ essential oil } & Lasioderma serricorne & [103] \\
\hline & & Rhizopertha dominica & \multirow{2}{*}{ [104] } \\
\hline & & Tribolium castaneum & \\
\hline Laggera pterodonta & essential oil & Lasioderma serricorne & [105] \\
\hline Lavandula angustifolia & essential oil & Sitophilus granarius & [106] \\
\hline \multirow{2}{*}{ Lavandula officinalis } & \multirow{2}{*}{ essential oil } & Sitophilus oryzae & \multirow{2}{*}{ [92] } \\
\hline & & Tribolium castaneum & \\
\hline \multirow{3}{*}{ Lavandula stoechas } & \multirow{3}{*}{ essential oil } & Lasioderma serricorne & \multirow{3}{*}{ [95] } \\
\hline & & Rhizopertha dominica & \\
\hline & & Tribolium castaneum & \\
\hline Lepidoploa aurea & ethanolic extract & Tenebrio molitor & [44] \\
\hline Litsea cubeba & essential oil & Lasioderma serricorne & [107] \\
\hline \multirow{2}{*}{ Litsea salicifolia } & \multirow{2}{*}{ essential oil } & Sitophilus zeamais & \multirow{2}{*}{ [108] } \\
\hline & & Tribolium castaneum & \\
\hline Linium usitatissium & essential oil & Cryptolestes ferrugineus & [43] \\
\hline Lippia javanica & essential oil & Sitophilus zeamais & [109] \\
\hline Lippia sidoides & essential oil & Tenebrio molitor & {$[110]$} \\
\hline \multirow{2}{*}{ Liriope muscari } & \multirow{2}{*}{ essential oil } & Lasioderma serricorne & \multirow{2}{*}{ [111] } \\
\hline & & Tribolium castaneum & \\
\hline Maytenus emarginata & ether extract & Oryzaephilus surinamensis & {$[70]$} \\
\hline Melia azedarach & essential oil & Cryptolestes ferrugineus & {$[43]$} \\
\hline \multirow{4}{*}{ Mentha piperita } & powder & Oryzaephilus surinamensis & {$[47]$} \\
\hline & \multirow{2}{*}{ essential oil } & Tribolium castaneum & \multirow{2}{*}{ [112] } \\
\hline & & Lasioderma serricorne & \\
\hline & essential oil & Sitophilus oryzae & {$[113]$} \\
\hline \multirow{2}{*}{ Mentha longifolia } & essential oil & Tribolium castaneum & {$[42]$} \\
\hline & essential oil & Sitophilus zeamais & [114] \\
\hline \multirow{3}{*}{ Mentha pulegium } & essential oil & Sitophilus granarius & [115] \\
\hline & \multirow{2}{*}{ essential oil } & Tribolium castaneum & \multirow{2}{*}{ [116] } \\
\hline & & Lasioderma serricorne & \\
\hline Mentha. & essential oil & Sitophilus oryzae & [85] \\
\hline \multirow{3}{*}{ Mesua ferrea } & water extract & & \\
\hline & ethanol extract & Oryzaephilus surinamensis & [68] \\
\hline & acetone extract & & \\
\hline Micromeria fruticosa & essential oil & Sitophilus granarius & [117] \\
\hline Minthostachys verticillata & essential oil & Sitophilus zeamais & [118] \\
\hline
\end{tabular}


Table 1. Cont.

\begin{tabular}{|c|c|c|c|}
\hline \multicolumn{4}{|c|}{ Coleoptera } \\
\hline Plant Species & Formulation & Pest Species & Reference \\
\hline \multirow{2}{*}{ Mosla soochowensis } & \multirow{2}{*}{ essential oil } & Sitophilus zeamais & \multirow{2}{*}[119]{} \\
\hline & & Tribolium confusum & \\
\hline \multirow{3}{*}{ Myristica fragrans } & \multirow{3}{*}{ essential oil } & Tribolium castaneum & \multirow{3}{*}[64]{} \\
\hline & & Sitophilus oryzae & \\
\hline & & Rhizopertha dominica & \\
\hline Myrtus communis & essential oil & Sitophilus oryzae & [57] \\
\hline \multirow{2}{*}{ Nardostachys chinensis } & essential oil & \multirow{2}{*}{$\begin{array}{l}\text { Tribolium castaneum Lasioderma } \\
\text { serricorne }\end{array}$} & \multirow{2}{*}[120]{} \\
\hline & supercritical $\mathrm{CO}_{2}$ fluid extract & & \\
\hline \multirow{3}{*}{ Nigella sativa } & \multirow{3}{*}{ essential oil } & Tribolium castaneum & \multirow{3}{*}{ [64] } \\
\hline & & Sitophilus oryzae & \\
\hline & & Rhizopertha dominica & \\
\hline \multirow{5}{*}{ Ocimum basilicum } & essential oil & Sitophilus oryzae & {$[121]$} \\
\hline & \multirow{4}{*}{ essential oil } & Sitophilus zeamais & {$[79]$} \\
\hline & & Tribolium castaneum & \multirow{3}{*}{ [122] } \\
\hline & & Tribolium confusum & \\
\hline & & Trogoderma granarium & \\
\hline \multirow{4}{*}{ Ocimum gratissimum } & \multirow{4}{*}{ essential oil } & Oryzaephilus surinamensis & \multirow{4}{*}[123]{} \\
\hline & & Rhizopertha dominica & \\
\hline & & Sitophilus oryzae & \\
\hline & & Tribolium castaneum & \\
\hline \multirow{2}{*}{ Origanum acutidens } & \multirow{2}{*}{ essential oil } & Lasioderma serricorne & \multirow{2}{*}{ [124] } \\
\hline & & Sitophilus granarius & \\
\hline Origanum majorana & essential oil & Tribolium confusum & \\
\hline Origanum minutiflorum & essential oil & Tribolium confusum & [125] \\
\hline Origanum onites & essential oil & Tribolium confusum & \\
\hline Origanum syriacum & essential oil & Tribolium confusum & \\
\hline \multirow{2}{*}{ Origanum vulgare } & essential oil & Sitophilus oryzae & {$[57]$} \\
\hline & essential oil & Tribolium castaneum & {$[46,125]$} \\
\hline Ostericum viridiflorum & essential oil & Tribolium castaneum & {$[126]$} \\
\hline Petroselinum crispum & essential oil & Sitophilus zeamais & {$[53]$} \\
\hline Perilla frutescens & essential oil & Lasioderma serricorne & {$[127]$} \\
\hline Pimenta dioica & powder & Oryzaephilus surinamensis & [47] \\
\hline Pimpinella anisum & essential oil & Tribolium castaneum & [46] \\
\hline Pinus longifolia & essential oil & Sitophilus oryzae & [82] \\
\hline Pituranthos tortuosus & essential oil & Sitophilus oryzae & {$[57]$} \\
\hline \multirow{2}{*}{ Platycladus orientalis } & \multirow{2}{*}{ essential oil } & Sitophilus oryzae & \multirow{2}{*}{ [128] } \\
\hline & & Tribolium castaneum & \\
\hline & water extract & & \\
\hline Pongamia pinnata & ethanol extract & Oryzaephilus surinamensis & [68] \\
\hline & acetone extract & & \\
\hline
\end{tabular}


Table 1. Cont.

\begin{tabular}{|c|c|c|c|}
\hline \multicolumn{4}{|c|}{ Coleoptera } \\
\hline Plant Species & Formulation & Pest Species & Reference \\
\hline \multirow{2}{*}{ Psidium guajava } & powder & \multirow{2}{*}{ Tribolium castaneum } & \multirow{2}{*}{ [75] } \\
\hline & ethanol extract & & \\
\hline Pulicaria gnaphalodes & essential oil & Tribolium castaneum & [42] \\
\hline Punica gronatum & ether extract & Oryzaephilus surinamensis & [70] \\
\hline \multirow{3}{*}{ Ricinus communis } & \multirow{2}{*}{ essential oil } & Tribolium castaneum & \multirow{2}{*}{ [116] } \\
\hline & & Lasioderma serricorne & \\
\hline & essential oil & Tribolium confusum & [74] \\
\hline Rosmarinus officinalis & essential oil & Tribolium confusum & [74] \\
\hline Salvia officinalis & powder & Oryzaephilus surinamensis & [47] \\
\hline Salvertia convallariaeodora & ether extract & Oryzaephilus surinamensis & [70] \\
\hline Sasurrea costus & essential oil & Cryptolestes ferrugineus & [43] \\
\hline Satureja hortensis & essential oil & Tribolium castaneum & [129] \\
\hline Schinus molle & \multirow{2}{*}{ essential oil } & \multirow{2}{*}{ Sitophilus oryzae } & \multirow{2}{*}{ [57] } \\
\hline Schinus terebinthifolius & & & \\
\hline Solanum nigrum & glycoalkaloid extract & Tenebrio molitor & [130] \\
\hline \multirow{3}{*}{ Syzygium aromaticum } & \multirow{3}{*}{ essential oil } & Tenebrio molitor & [71] \\
\hline & & Sitophilus oryzae & \multirow{2}{*}{ [92] } \\
\hline & & Tribolium castaneum & \\
\hline \multirow{2}{*}{ Syzygium cumini } & essential oil & Tribolium confusum & {$[74]$} \\
\hline & essential oil & Sitophilus oryzae & [57] \\
\hline \multirow{3}{*}{$\begin{array}{c}\text { Tanacetum cinerariifolium } \\
\text { Tagetes erecta }\end{array}$} & \multirow{3}{*}{$\begin{array}{l}\text { essential oil } \\
\text { essential oil }\end{array}$} & Tribolium castaneum & \multirow{3}{*}{$\begin{array}{c}{[46]} \\
{[131]}\end{array}$} \\
\hline & & Sitophilus oryzae & \\
\hline & & Tribolium castaneum & \\
\hline \multirow{2}{*}{ Tagetes minuta } & \multirow{2}{*}{ essential oil } & Sitophilus oryzae & \multirow{2}{*}{ [131] } \\
\hline & & Tribolium castaneum & \\
\hline \multirow{2}{*}{ Tagetes patula } & \multirow{2}{*}{ essential oil } & Sitophilus oryzae & \multirow{2}{*}{ [131] } \\
\hline & & Tribolium castaneum & \\
\hline Teucrium polium & essential oil & Tribolium castaneum & [132] \\
\hline Thespesia populnea var. acutiloba & methanol extract & Trogoderma granarium & [63] \\
\hline Thuja occidentalis & essential oil & Sitophilus oryzae & [57] \\
\hline \multirow{3}{*}{ Trewia nudiflora } & water extract & \multirow{3}{*}{ Oryzaephilus surinamensis } & \multirow{3}{*}[68]{} \\
\hline & ethanol extract & & \\
\hline & acetone extract & & \\
\hline & water extract & & \\
\hline Typhonium trilobatum & ethanol extract & Oryzaephilus surinamensis & [68] \\
\hline & acetone extract & & \\
\hline Valeriana jatamansi & essential oil & Tribolium castaneum & [120] \\
\hline & supercritical $\mathrm{CO}_{2}$ fluid extract & Lasioderma serricorne & \\
\hline Valeriana officinalis & essential oil & Tribolium castaneum Lasioderma & [120] \\
\hline & supercritical $\mathrm{CO}_{2}$ fluid extract & serricorne & \\
\hline Vepris heterophylla & essential oil & Sitophilus oryzae & [133] \\
\hline
\end{tabular}


Table 1. Cont

\begin{tabular}{|c|c|c|c|}
\hline \multicolumn{4}{|c|}{ Coleoptera } \\
\hline Plant Species & Formulation & Pest Species & Reference \\
\hline \multirow{2}{*}{$\begin{array}{l}\text { Verbascum cheiranthifolium } \\
\text { Verbascum speciosum }\end{array}$} & ethanol extract & Sitophilus oryzae & [134] \\
\hline & ethanol extract & Sitophilus oryzae & [134] \\
\hline Viola odorata & essential oil & Cryptolestes ferrugineus & [43] \\
\hline Vitex negundo & ether extract & Oryzaephilus surinamensis & {$[70]$} \\
\hline Xylopia aethiopica & essential oil & Sitophilus oryzae & [87] \\
\hline \multirow{2}{*}{ Zanthoxylum armatum } & \multirow{2}{*}{ essential oil } & Lasioderma serricorne & \multirow{2}{*}{ [135] } \\
\hline & & Tribolium castaneum & \\
\hline \multirow{2}{*}{ Zanthoxylum dissitum } & \multirow{2}{*}{ essential oil } & Lasioderma serricorne & \multirow{2}{*}{ [135] } \\
\hline & & Tribolium castaneum & \\
\hline $\begin{array}{l}\text { Zanthoxylum planispinum var. } \\
\text { dintanensis }\end{array}$ & essential oil & $\begin{array}{c}\text { Tribolium castaneum Lasioderma } \\
\text { serricorne }\end{array}$ & [136] \\
\hline Zingiber officinale & essential oil & Sitophilus oryzae & [85] \\
\hline \multirow{2}{*}{ Zingiber purpureum } & essential oil & Lasioderma serricorne & \multirow{2}{*}{ [137] } \\
\hline & essential oil & Tribolium castaneum & \\
\hline \multicolumn{4}{|c|}{ Lepidoptera } \\
\hline Plant Species & Formulation & Pest Species & Reference \\
\hline Acacia nilotica & acetone and pet ether extracts & Corcyra cephalonica & [138] \\
\hline Acorus calamus & $\begin{array}{c}\text { petroleum ether extract } \\
\text { acetone extract }\end{array}$ & Sitotroga cerealella & [139] \\
\hline Aframomum melegueta & $\begin{array}{c}\text { powder } \\
\text { extract }\end{array}$ & Sitotroga cerealella & [140] \\
\hline Agastache rugosa & plant extract & Plodia interpunctella & [46] \\
\hline Ajugaiva & methanol extract & Plodia interpunctella & [141] \\
\hline Allium cepa & essential oil & Plodia interpunctella & [142] \\
\hline & essential oil & Sitotroga cerealella & [143] \\
\hline \multirow{5}{*}{ Allium sativum } & $\begin{array}{l}\text { major component: diallyl } \\
\text { disulfide and diallyl trisulfide }\end{array}$ & Sitotroga cerealella & [143] \\
\hline & $\begin{array}{l}\text { major component: diallyl } \\
\text { trisulfide }\end{array}$ & Sitotroga cerealella & {$[144-146]$} \\
\hline & essential oil & Ephestia kuehniella & [147-149] \\
\hline & essential oil & Ephestia cautella & [149] \\
\hline & essential oil & Plodia interpunctella & [147] \\
\hline \multirow{2}{*}{ Anacardium occidentale } & essential oil & Ephestia cautella & [150] \\
\hline & $\begin{array}{l}\text { ethanolic oil extract and } \\
\text { petroleum ether extract }\end{array}$ & Ephestia cautella & {$[151]$} \\
\hline \multirow{2}{*}{ Anethum graveolens } & essential oil & Plodia interpunctella & [152] \\
\hline & $\begin{array}{c}\text { oils applied as a } 30 \% \text { aqueous } \\
\text { solution }\end{array}$ & Ephestia kuehniella & [153] \\
\hline Arachis hypogaea & acetone extract & Corcyra cephalonica & [154] \\
\hline Armoracia rusticana & essential oil & Plodia interpunctella & [54] \\
\hline Artemisia haussknechtii & essential oil & Ephestia kuehniella & [155] \\
\hline Artemisia herba alba & essential oil & Ephestia kuehniella & [156] \\
\hline \multirow{2}{*}{ Artemisia khorassanica } & essential oil & Sitotroga cerealella & [157] \\
\hline & essential oil & Plodia interpunctella & [158] \\
\hline Artemisia sieberi Bess & essential oil & Sitotroga cerealella & [157] \\
\hline \multirow{2}{*}{ Artemisia vulgaris } & essential oil & Cadra cautella & [159] \\
\hline & essential oil & Sitotroga cerealella & [58] \\
\hline \multirow{2}{*}{$\begin{array}{c}\text { Azadirachta indica } \\
\text { Azadirachta indica }+ \text { Bacilus } \\
\text { thuringiensis }\end{array}$} & essential oil & Ephestia cautella & [160] \\
\hline & & Ephestia cautella & [160] \\
\hline
\end{tabular}


Table 1. Cont.

\begin{tabular}{|c|c|c|c|}
\hline \multicolumn{4}{|c|}{ Lepidoptera } \\
\hline Plant Species & Formulation & Pest Species & Reference \\
\hline Betula lenta & essential oil & $\begin{array}{l}\text { Plodia interpunctella } \\
\text { Ephestia kuehniella }\end{array}$ & [147] \\
\hline Brassica alba & essential oil & Ephestia cautella & [148] \\
\hline Callistemon viminalis & essential oil & Ephestia kuehniella & [161] \\
\hline Calotripis procera roots & extract & Cadra cautella & [162] \\
\hline Capsicum annuum & plant extract & Plodia interpunctella & [46] \\
\hline \multirow{2}{*}{ Capsicum frutescens } & essential oil & Ephestia cautella & [150] \\
\hline & $\begin{array}{l}\text { ethanolic oil extract and } \\
\text { petroleum ether extract }\end{array}$ & Ephestia cautella & [151] \\
\hline Carum copticum & essential oil & $\begin{array}{l}\text { Ephestia kuehniella } \\
\text { Plodia interpunctella }\end{array}$ & [163] \\
\hline Cassia angustifolia & protease inhibitor & Plodia interpunctella & [164] \\
\hline Centaurium erythraea & methanol extract & Plodia interpunctella & [141] \\
\hline Cinnamomum camphora & essential oil & Ephestia kuehniella & [165] \\
\hline Cinnamomum. & $\begin{array}{l}\text { oil encapsulated with } \\
\text { different types of alcohol }\end{array}$ & Plodia interpunctella & [166] \\
\hline \multirow[b]{3}{*}{ Cinnamomum zeylanicum } & $\begin{array}{l}\text { polyethylene-laminated } \\
\text { polypropylene films }\end{array}$ & Plodia interpunctella & [167] \\
\hline & essential oil & Plodia interpunctella & {$[168,169]$} \\
\hline & $\begin{array}{l}\text { essential oil } \\
\text { essential oil }\end{array}$ & $\begin{array}{l}\text { Plodia interpunctella } \\
\text { Ephestia kuehniella }\end{array}$ & [147] \\
\hline Cistus ladanifer & methanol extract & Plodia interpunctella & \\
\hline Cistus monspeliensis & methanol extract & Plodia interpunctella & [141] \\
\hline Cistus salviaefolius & methanol extract & Plodia interpunctella & \\
\hline Citrus aurantium & essential oil & Sitotroga cerealella & [170] \\
\hline Citrus bergamia & essential oil & Plodia interpunctella & [171] \\
\hline \multirow[t]{2}{*}{ Citrus limon } & essential oil & $\begin{array}{l}\text { Plodia interpunctella } \\
\text { Ephestia kuehniella }\end{array}$ & [172] \\
\hline & essential oil & Ephestia kuehniella & [173] \\
\hline Citrus reticulata & plant extract & Plodia interpunctella & [46] \\
\hline Citrus vulgaris & essential oil & Ephestia kuehniella & [155] \\
\hline \multirow[t]{2}{*}{ Clausena anisata } & essential oil & Sitotroga cerealella & [174] \\
\hline & root bark powder & Plodia interpunctella & \\
\hline \multirow[t]{2}{*}{ Cleisthopholis patens } & stem bark powder & Plodia interpunctella & [175] \\
\hline & leaf powder & Plodia interpunctella & \\
\hline \multirow[t]{2}{*}{ Conocarpus lancifolius (leaves) } & aqueous and ethanolic extract & Ephestia cautella & [176] \\
\hline & essential oil & Sitotroga cerealella & [177] \\
\hline \multirow{3}{*}{ Coriandrum sativum } & essential oil & Ephestia kuehniella & {$[178,179]$} \\
\hline & essential oil & Plodia interpunctella & [178] \\
\hline & essential oil & Corcyra cephalonica & [82] \\
\hline Cuтіпит сутіпит & essential oil & Ephestia kuehniella & [180] \\
\hline Cupressus lusitanica & leaf essential oils & Sitotroga cerealella & [181] \\
\hline Cupressus sempervirens & essential oil & Cadra cautella & [159] \\
\hline $\begin{array}{c}\text { Cupressus sempervirens } L . \\
\text { horizontalis }\end{array}$ & resin essential oil & Ephestia kuehniella & [182] \\
\hline Cymbopogon martinii & $\begin{array}{c}\text { essential oil } \\
\text { sesquiterpene lactones }\end{array}$ & Plodia interpunctella & [171] \\
\hline Cyrtocymura cincta & $\begin{array}{l}\text { isolated from leaves and } \\
\text { flowers }\end{array}$ & Sitotroga cerealella & [183] \\
\hline \multirow{3}{*}{$\begin{array}{c}\text { Dalbergia sissoo } \\
\text { Dimorphandra mollis } \\
\text { Dryopteris filix (mas root and } \\
\text { rhizome) }\end{array}$} & acetone extract & Corcyra cephalonica & [138] \\
\hline & flowers & Sitotroga cerealella & [184] \\
\hline & ethanolic extract & Corcyra cephalonica & [185] \\
\hline Elaeagnus angustifolia & $\begin{array}{l}\text { ethyl acetate } \\
\text { aqueous }\end{array}$ & $\begin{array}{l}\text { Ephestia cautella } \\
\text { Ephestia cautella }\end{array}$ & [186] \\
\hline Elettaria cardamomum & essential oil & Ephestia kuehniella & {$[187,188]$} \\
\hline
\end{tabular}


Table 1. Cont

\begin{tabular}{|c|c|c|c|}
\hline \multicolumn{4}{|c|}{ Lepidoptera } \\
\hline Plant Species & Formulation & Pest Species & Reference \\
\hline \multirow[t]{2}{*}{ Eucalyptus astringens } & essential oil & $\begin{array}{l}\text { Ephestia cautella } \\
\text { Ephestia kuehniella }\end{array}$ & [189] \\
\hline & essential oil & $\begin{array}{l}\text { Ephestia kuehniella } \\
\text { Ephestia cautella }\end{array}$ & [180] \\
\hline Eucalyptus camaldulensis & essential oil & $\begin{array}{l}\text { Ephestia kuehniella } \\
\text { Cadra cautella }\end{array}$ & [189] \\
\hline Eucalyptus dives & oil and constituents & Plodia interpunctella & [190] \\
\hline \multirow[t]{2}{*}{ Eucalyptus globulus } & essential oil & $\begin{array}{l}\text { Ephestia cautella } \\
\text { Ephestia kuehniella }\end{array}$ & [149] \\
\hline & essential oil & Plodia interpunctella & [171] \\
\hline Eucalyptus lehmannii & essential oil & $\begin{array}{l}\text { Ephestia cautella } \\
\text { Ephestia kuehniella }\end{array}$ & [189] \\
\hline Eucalyptus leucoxylon & essential oil & $\begin{array}{l}\text { Ephestia cautella } \\
\text { Ephestia kuehniella }\end{array}$ & [189] \\
\hline Eucalyptus obliqua & essential oil & Corcyra cephalonica & [82] \\
\hline Eucalyptus platyphylla & essential oil & Ephestia cautella & [191] \\
\hline Eucalyptus rudis & essential oil & $\begin{array}{l}\text { Ephestia cautella } \\
\text { Ephestia kuehniella }\end{array}$ & [189] \\
\hline Eucalyptus saligna & leaf essential oils & Sitotroga cerealella & [181] \\
\hline Ferula galbaniflua & essential oil & Cadra cautella & [159] \\
\hline Ferula gummosa & essential oil & Ephestia kuehniella & {$[161,188]$} \\
\hline Geranium maculatum & essential oil & Plodia interpunctella & {$[171,192]$} \\
\hline Ginkgo biloba & plant extract & Plodia interpunctella & [46] \\
\hline Glossocardia bosvallia & hexane extracts & Corcyra cephalonica & [193] \\
\hline Hyossopus officinalis & essential oil & Plodia interpunctella & [152] \\
\hline Hypericum scabrum & essential oil & Ephestia kuehniella & [117] \\
\hline Hyssoppus officinalis & essential oil & Ephestia kuehniella & [117] \\
\hline Lantana camara & essential oil & Cadra cautella & [194] \\
\hline Launaea arborescens & $\begin{array}{l}\text { methanol extract } \\
\text { essential oil }\end{array}$ & $\begin{array}{l}\text { Plodia interpunctella } \\
\text { Ephestia kuehniella }\end{array}$ & $\begin{array}{l}{[141]} \\
{[195]}\end{array}$ \\
\hline Laurus nobilis & essential oil & $\begin{array}{l}\text { Plodia interpunctella } \\
\text { Ephestia kuehniella }\end{array}$ & [172] \\
\hline Lavandula angustifolia & essential oil & Ephestia kuehniella & [196] \\
\hline Lavandula angustifolia & extract & Ephestia kuehniella & [197] \\
\hline Lavandula angustifolia & essential oil & Plodia interpunctella & [171] \\
\hline Lavandula dentata & methanol extract & Plodia interpunctella & [141] \\
\hline Lawsonia inermis & pet ether extract & Corcyra cephalonica & [138] \\
\hline Lippia turbinata & essential oil & Plodia interpunctella & [198] \\
\hline Maxillaria tenuifolia & $\begin{array}{l}\text { ethanolic oil extract and } \\
\text { petroleum ether extract }\end{array}$ & Ephestia cautella & [151] \\
\hline \multirow{3}{*}{$\begin{array}{l}\text { Melaleuca viridiflora } \\
\text { Mentha longifolia }\end{array}$} & essential oil & Cadra cautella & [159] \\
\hline & essential oil & Ephestia kuehniella & [199] \\
\hline & essential oil & Corcyra cephalonica & [113] \\
\hline \multirow{3}{*}{ Mentha piperita } & polylactic acid solution & Plodia interpunctella & [200] \\
\hline & essential oil & Plodia interpunctella & {$[171,192]$} \\
\hline & essential oil & Ephestia cautella & [191] \\
\hline Mentha pulegium & essential oil & Ephestia kuehniella & [201] \\
\hline Mentha spicata & essential oil & $\begin{array}{l}\text { Plodia interpunctella } \\
\text { Ephestia kuehniella }\end{array}$ & [202] \\
\hline Micromere fruticosa & essential oil & Ephestia kuehniella & {$[117,203]$} \\
\hline Monodora tenuifolia & essential oil & Ephestia cautella & [150] \\
\hline \multirow{3}{*}{ Myristica fragrans } & essential oil & Cadra cautella & [159] \\
\hline & essential oil & Plodia interpunctella & [204] \\
\hline & essential oil & Ephestia kuehniella & {$[195,205]$} \\
\hline Myrtus commonis & essential oil & $\begin{array}{l}\text { Plodia interpunctella } \\
\text { Ephestia kuehniella }\end{array}$ & [172] \\
\hline Nepata racemosa & essential oil & Ephestia kuehniella & [203] \\
\hline Neroli birgard & essential oil & Sitotroga cerealella & [170] \\
\hline
\end{tabular}


Table 1. Cont.

\begin{tabular}{|c|c|c|c|}
\hline \multicolumn{4}{|c|}{ Lepidoptera } \\
\hline Plant Species & Formulation & Pest Species & Reference \\
\hline \multirow{3}{*}{ Ocimum basilicum } & $\begin{array}{c}\text { oils applied as a } 30 \% \text { aqueous } \\
\text { solution }\end{array}$ & Ephestia kuehniella & [153] \\
\hline & essential oil & Ephestia kuehniella & {$[178,201,202]$} \\
\hline & essential oil & Plodia interpunctella & {$[178,202]$} \\
\hline \multirow{2}{*}{ Ocimum kilimandscharicum } & ground leaves & Sitotroga cerealella & [206] \\
\hline & essential oil & Sitotroga cerealella & \\
\hline Ocimum suave & $\begin{array}{c}\text { essential oil } \\
\text { dry or ground leaves }\end{array}$ & Sitotroga cerealella & [207] \\
\hline \multirow[t]{2}{*}{ Origanum acutidens } & essential oil & Ephestia kuehniella & {$[117,124]$} \\
\hline & essential oil & Corcyra cephalonica & [208] \\
\hline \multirow[t]{2}{*}{ Origanum majorana } & essential oil & $\begin{array}{l}\text { Plodia interpunctella } \\
\text { Ephestia kuehniella }\end{array}$ & [172] \\
\hline & essential oil & Ephestia kuehniella & [173] \\
\hline Origanum onites & essential oil & $\begin{array}{l}\text { Plodia interpunctella } \\
\text { Ephestia kuehniella }\end{array}$ & [205] \\
\hline Origanum syriacum var. bevanii & essential oil & Ephestia kuehniella & [180] \\
\hline \multirow{2}{*}{ Origanum vulgare } & essential oil & Plodia interpunctella & [46] \\
\hline & essential oil & Ephestia kuehniella & {$[156,203]$} \\
\hline Origanum vulgaris & essential oil & Ephestia kuehniella & [209] \\
\hline Parthenium hysterophorus & acetone extract & Corcyra cephalonica & [138] \\
\hline Peganum harmala & methanol extract & Plodia interpunctella & [141] \\
\hline Petroselinum crispum & essential oil & Ephestia kuehniella & [179] \\
\hline \multirow[t]{2}{*}{ Petroselinum sativum } & essential oil & Plodia interpunctella & [152] \\
\hline & essential oil & Ephestia cautella & [149] \\
\hline \multirow[t]{2}{*}{ Pimpinella anisum } & essential oil & Ephestia kuehniella & {$[147,149,180]$} \\
\hline & essential oil & Plodia interpunctella & {$[46,147]$} \\
\hline Pinus brutia & essential oil & Ephestia kuehniella & [210] \\
\hline Pinus densiflora & diterpene resin acids (DRAs) & Plodia interpunctella & [211] \\
\hline Pinus longifolia & essential oil & Corcyra cephalonica & [82] \\
\hline Pinus pinea & essential oil & Ephestia kuehniella & [210] \\
\hline Piper nigrum & essential oil & Corcyra cephalonica & [113] \\
\hline Piper sarmentosum & hexane extracts & Plodia interpunctella & [212] \\
\hline Pistacia lentiscus & essential oil & Ephestia kuehniella & [213] \\
\hline Polyalthia longifolia & essential oil & Sitotroga cerealella & {$[174]$} \\
\hline \multirow{2}{*}{ Portulaca oleracea } & essential oil & Ephestia cautella & [214] \\
\hline & essential oil & Ephestia kuehniella & [215] \\
\hline Prunus & grain coating with amygdalin & Plodia interpunctella & [216] \\
\hline Psychotria prunifolia & leaves & Sitotroga cerealella & [184] \\
\hline \multirow{2}{*}{ Ricinus communis } & essential oil & Ephestia cautella & {$[150,214]$} \\
\hline & $\begin{array}{l}\text { ethanolic oil extract and } \\
\text { petroleum ether extract }\end{array}$ & Ephestia cautella & [151] \\
\hline \multirow{5}{*}{ Rosemarinus officinalis } & essential oil & Ephestia kuehniella & {$[149,180,196]$} \\
\hline & essential oil & Ephestia cautella & [149] \\
\hline & essential oil & Cadra cautella & [159] \\
\hline & essential oil & Plodia interpunctella & [152] \\
\hline & methanol extract & Plodia interpunctella & [141] \\
\hline Ruta graveolens & essential oil & Ephestia kuehniella & [201] \\
\hline Ruta montana & essential oil & Ephestia kuehniella & [156] \\
\hline Salvia limbata & essential oil & Ephestia kuehniella & [117] \\
\hline Salvia nemorosa & essential oil & Ephestia kuehniella & [117] \\
\hline \multirow{2}{*}{ Salvia officinalis } & essential oil & Ephestia kuehniella & [188] \\
\hline & polylactic acid solution & Plodia interpunctella & [200] \\
\hline \multirow{3}{*}{ Satureja hortensis } & & Plodia interpunctella & [129] \\
\hline & essential oil & Ephestia kuehniella & [129] \\
\hline & essential oil & Cadra cautella & [159] \\
\hline \multirow{2}{*}{ Satureja thymbra } & & Plodia interpunctella & [205] \\
\hline & essential oil & Ephestia kuehniella & {$[172,205]$} \\
\hline Satureje hortensis & essential oil & Ephestia kuehniella & [117] \\
\hline Syzygium aromaticum & essential oil & Ephestia kuehniella & [165] \\
\hline
\end{tabular}


Table 1. Cont.

\begin{tabular}{cccc}
\hline & & Lepidoptera & \\
Plant Species & Formulation & Pest Species & Reference \\
\hline Tanacetum cinerariifolium & essential oil & Plodia interpunctella & {$[46]$} \\
Thymus daenensis & essential oil & Plodia interpunctella & Ephestia kuehniella \\
& essential oil & Plodia interpunctella & {$[152]$} \\
Thymus vulgaris & essential oil & Ephestia kuehniella & {$[117]$} \\
Tithonia diversifolia & leaves & Sitotroga cerealella & {$[184]$} \\
Trachyspermum ammi & essential oil & Plodia interpunctella & {$[204]$} \\
Trigonella foenum-graecum & protease inhibitor & Plodia interpunctella & {$[164]$} \\
Vitex pseudo-negundo & essential oil & Plodia interpunctella & {$[158]$} \\
Xylopia aethiopica & essential oil & Ephestia cautella & {$[150]$} \\
& ethanolic oil extract and & Ephestia cautella & {$[151]$} \\
Zingiber officinale & petroleum ether extract & Ephestia kuehniella & {$[153]$} \\
Ziziphora clinopodioides Lam. & essential oil & Ephestia kuehniella & {$[218]$} \\
\hline
\end{tabular}

\subsection{Insects (Insecta)}

Pests affecting grains can be found in various orders of insects. Some of these belong to the major pests affecting stored crops and food, whereas some are of minor importance. Among the most important ones are beetles (Coleoptera), true bugs (Hemiptera), butterflies and moths (Lepidoptera). Therefore, we will focus on these three orders.

\subsubsection{Beetles (Coleoptera)}

Coleopterans form the largest order within the animal kingdom, with more than 400,000 species and about 200 families. It is not surprising that within this order we may find pests affecting grains, which are of economic importance. One of the largest families of Coleoptera is Tenebrionidae, or as they are commonly known, darkling beetles. Within this family there are some very important grain pests. Firstly, there is the confused flour beetle Tribolium confusum Jacq. Du Val, a pest that feeds on all kinds of grains and flour. T. confusum can have as many as five generations per year and every female can lay as many as 600 eggs on the stored product. In the case of T. confusum, very few botanical insecticides have been tested. Firstly, Carum copticum and Cuminum cyminum were effective in their contact toxicity effects against $T$. confusum with $\mathrm{LD}_{50}$ values of $0.037 \mu \mathrm{g} / \mathrm{mg}$ and $0.039 \mu \mathrm{g} / \mathrm{mg}$, respectively [65]. Secondly, Aster ageratoides [60], Juniperus polycarpos and Juniperus sabina [100] acted as fumigants in the form of EO, whereas Crithmum maritimum did not cause any mortality [83].

Tribolium castaneum Herbst, commonly known as the rust-red flour beetle, belongs to the same genus as T. confusum, with which it has physical similarities, life cycle and feeding habits, meaning it is also a secondary pest. T. castaneum, as a model beetle species, has been the most tested beetle in regard to the effects of botanical insecticides. The tested natural substances have been mainly evaluated for their fumigant toxicity, contact toxicity or repellant properties. Tagetes minuta [131], Tagetes patula [131], Platycladus orientalis (fruits) [128], A. sativum [49] and M. piperita [112] have been proven to be the most effective fumigants against the adults of this species. Eucalyptus procera [97], basil [79], orange [79] and Satureja hortensis oils were toxic after contact with T. confusum adults [129], whereas Citrus reticulata and Citrus sinensis essential oils were effective against larvae [76]. Finally, many plant extracts were proven to be effective as repellents; the repellency abilities of the botanical substances were assessed based on the area test, as described by McDonald et al. (1970) [220]—namely, Litsea salicifolia [108], Artemisia anethoides [55], Zanthoxylum planispinum [136] and Hyptis suaveolens exhibited repellency rates of more 
than $90 \%$ [98]. C. reticulata was also effective as a repellent in the form of powder of ethanol extract.

The yellow mealworm beetle, Tenebrio molitor L., is also a secondary pest, feeding on flour, grains and plant-based products. Garlic essential oil caused necrosis in larvae, pupae and adults of T. molitor L. after 20-40 h of exposure [48], whereas Bidens sulphurea and Adenocalymma nodosum were more effective against the pupae [44]. Cinnamon oil was most toxic to T. molitor L. larvae, whereas clove oil was most effective on adults. Cinnamon oil mainly yielded eugenol (10.19\%), trans-3-caren-2-ol (9.92\%) and benzyl benzoate $(9.68 \%)$; whereas clove oil yielded eugenol $(26.64 \%)$, caryophyllene $(23.73 \%)$ and caryophyllene oxide (17.74\%) [71]. Interestingly, some research on T. molitor L. has focused on the sublethal effects of glycoalkaloids, describing malformations of organelles, chromatin condensation and altered contractility of the heart and oviduct [130].

Another large family of Coleoptera is the Curculionidae, or as they are commonly known, "snout beetles". In this family there are 83,000 species described worldwide. There are some important grain pests in this family. Sitophilus granarius L. is a frequent pest of wheat and barley, but also can attack other cereals, such as maize, sorghum and rice. Lower developmental stages (eggs, larvae and pupae) of $S$. granarius complete their development inside a seed kernel or products, which is common for all other primary insects. Every female of the species can produce as many as 400 eggs, which are placed separately inside the seeds. It is a primary pest which feeds on all Poaceae grains. Many plant extracts have been tested for acute toxicity against $S$. granarius with Calendula officinalis being amongst the most promising contact toxicant extracts [67], whereas Origanum acutidens [124], C. maritimum [83] and L. angustifolia were the best fumigants [106]. Furthermore, L. angustifolia [106], C. copticum and Cuminum cyminum were very powerful in terms of their contact toxicity [65]; L. angustifolia was also effective as a repellent [106].

The rice weevil (Sitophilus oryzae L.) is morphologically very similar to S. granarius, but it is more commonly found in stored rice. It feeds mainly on cereal seeds and is less likely to feed on grain products. It is also more resistant to lower temperatures than S. granarius. Among the natural substances trialed for their fumigant properties, the most effective were the essential oils obtained from Cymbopogon citratus and Zingiber officinale [85], O. basilicum [121], Origanum vulgare and Citrus lemon oils [57]. Essentials oils of fennel (Foeniculum vulgare), caraway (C. carvi), cinnamon (Cinnamomum verum), citronella (Cymbopogon winterianus), nutmeg (Myristica fragrans) and black cumin (Nigella sativa) were all proven to be highly competent as contact toxicity insecticides [64]. Additionally, Artemisia judaica, Callistemon viminals and O. vulgare caused high-contact toxicity to $S$. oryzae with $\mathrm{LC}_{50}$ values of $0.08,0.09$ and $0.11 \mathrm{mg} / \mathrm{cm}^{2}$, respectively [57]. Ocimum gratissimum (EO) and its constituents [123], as well as H. suaveolens (EO), seemed to be very effective as repellents [98]. Finally, some botanical extracts (Acorus calamus + Corchorus capsularis seed petroleum ether extract, A. calamus + Thevetia neriifolia seed petroleum ether extract and A. calamus + Zingiber cassumunar Roxb. rhizome petroleum ether extract) were assessed in terms of the synergism of their toxic effects [45] or repellent effects (Hyptis spicigera, Vepris heterophylla) [133].

The maize weevil (Sitophilus zeamais Motsch) is another important pest, of an appearance similar the previously mentioned beetles; it is mainly found in stored maize but can sometimes be found in wheat and barley. S. zeamais Motsch has the ability to fly; hence, the infestation can start in the ripening crop in the field and can carry on in storage. Chenopodium ambrosioides [221] has been a very effective toxic fumigant, as has Blumea balsamifera [222], as well as orange and basil oil [79]. Kadsura heteroclite stems (EO) were the most effective in terms of contact toxicity, followed by Cayratia japonica (EO) [101]. Furthermore, L. salicifolia [108] and Anethum graveolens (EO), Petroselinum crispum (EO), F. vulgare (EO) and C. cyminum (EO) were effective repellents against the maize weevil [53].

There are also some important pests of grains within other families. For example, Lasioderma serricorne (Fabricius) (Anobiidae), commonly known as the "cigarette beetle", is a secondary pest, which feeds on a great variety of products, including grain-based products, 
herbs, and legumes. It can have up to four generations per year and it overwinters as larvae. Extracts of the botanical species O. acutidens seemed to be the most effective in terms of fumigant activities against this pest [124], followed by Artemisia mongolica [81], Alpinia blepharocalyx [50] and Perilla frutescens [127]. P. frutescens (L.) [127], Z. planispinum [127] and Artemisia stolonifera [59] extracts and ingredient components were also very promising as alternative and greener fumigants. Finally, M. piperita menthol extract showed very strong repellent properties, with $80 \%$ repellency after $4 \mathrm{~h}$ at the test concentration of $3.15 \mu \mathrm{L} / \mathrm{cm}^{2}$ [112], which was much greater than Z. planispinum essential oil, exhibiting $62 \%$ repellency after $4 \mathrm{~h}$ of exposure at $78.63 \mathrm{~nL} / \mathrm{cm}^{2}$ [136], and Laurus nobilis, exhibiting $60 \%$ repellency at a concentration of $0.2 \mu \mathrm{L} / \mathrm{cm}^{2}$ after $24 \mathrm{~h}$ of exposure [103]. Cryptolestes ferrugineus (Stephens) and other Cryptolestes species (Cucujidae) are important secondary pests of food grains [223]. EO from C. reticulata peel achieved 100\% fumigant mortality, contact toxicity that was fully effective $(99.0 \%$ after $48 \mathrm{~h}$ of exposure at the dosage of $2.0 \mu \mathrm{L} / \mathrm{cm}^{2}$ ) and finally had a very good repellency rate (achieved $>80.0 \%$ after $60 \mathrm{~h}$ of exposure at $0.4 \mu \mathrm{L} / \mathrm{cm}^{2}$ ) [77]. Unfortunately, according to other studies carried out with extracts of Melia azedarach, Linium usitatissium, Ferula narthex, Sasurrea costus, Viola odorata and Achyranthus aspera, they were not very effective, although M. azedarach was able to decrease larval and pupal emergence [122].

The saw-toothed grain beetle (Oryzaephilus surinamensis L. (Silvanidae)) feeds on grains, as well as bran and grain products. It is a secondary pest that lays eggs in flour and causes further qualitative and quantitative losses. Regarding the fumigant activity of O. gratissimum essential oil [123], which showed the best results at a concentration of $1 \mathrm{~mL} / \mathrm{L}$ air, the oil caused a mortality rate of $99 \% 24 \mathrm{~h}$ after treatment, respectively, followed by C. maritimum [83], Eucalyptus globules and L. stoechas [95]. The essential oils of H. suaveolens leaves [98], C. maritimum [83] and Artemisia herba-alba and Artemisia absinthium caused necrosis to adults [56]. Powdered Salvia officinalis also caused a high mortality rate, reaching $80 \%$ (the powdered plant was added to culture containers at concentrations of $5.88 \%$ ) [47]. Finally, Pongamia pinnata has the potential for use as a mediocre repellent [68]. Rhyzopertha dominica Fab. can be considered the main insect pest species in grains stored around the world [224,225]. It is a primary pest of stored products, feeding on all cereals and grain products. According to a study published in 2017 by Tawfeek et al., essential oils of fennel (F. vulgare), caraway (C. carvi), cinnamon (C. verum), citronella (C. winterianus), nutmeg (M. fragrans) and black cumin (N. sativa) seemed to be very active, with fennel being the most effective [64]. The essential oil of H. suaveolens is reported to have potential as a contact insecticide [98]. Lastly, L. nobilis and Eucalyptus floribundi are two essential oils with repellent and fumigant properties [94,189].

Khapra beetle (Trogoderma granarium Everts.) is the only genus from the family Dermestidae that feeds on plant-based products, especially stored grains. For many countries it is considered a quarantine concern because its spread occurs mainly through international trade. According to Derbalah and his team, in a research study in 2011, seven plant methanol extracts were tested for their insecticidal properties and the results were positive, with Cassia senna being the most effective $(100 \mathrm{mg} / \mathrm{L}$ achieved $86.7 \%$ mortality after one week) [63]. Interestingly, the plant extracts of Bauhinia purpurea, C. senna, Caesalpinia gilliesii, Cassia fistula, Chrysanthemum frutescens, Euonymus japonicus and Thespesia populnea var. acutiloba were also effective towards the beetle and displayed no side effects to rats, indicating friendliness to mammals. Finally, the essential oil of O. basilicum was found to cause mortality at $54.33 \%$ after $72 \mathrm{~h} \mathrm{[122].}$

As one can see, there are not enough data concerning the sublethal action of plantderived substances on beetles, such as the research on the sublethal effects of glycoalkaloids on T. molitor L. [130], as well as their mode of action (Table 1), compared to the research on other groups, especially moths and butterflies (see below). We think that basic research on these sublethal effects, including the analysis of both behavioral and physiological changes, may provide plenty of important data, which can be applied in studies leading to novel natural insecticides. 


\subsubsection{Butterflies and Moths (Lepidoptera)}

The range of lepidopteran species representing pests of grains and the research on plant-derived substances tested against them is wide (Table 1). The most important pests affecting grains are found within the Pyralidae family, although Sitotroga cerealella Oliv., belonging to Gelechiidae, is also considered a serious pest affecting grains.

S. cerealella Oliv. burrows in grains and makes them unfit for human consumption. The families Asteraceae, Bignoniaceae, Fabaceae and Rubiaceae contain species with insecticidal and repellent properties with potential applications against $S$. cerealella in stored grains. The dried ground leaves and essential oil of Ocimum kilimandscharicum exhibited significant activity against this species in maize and sorghum grains in the laboratory. In particular, there was no adult survival or progeny production in grains treated with each of the two materials at doses of $25.0 \mathrm{~g}$ (dried ground leaves) and $0.3 \mathrm{~g}$ (essential oil) per $250 \mathrm{~g}$ of grain, respectively. In addition, ground leaves and the essential oil protected the grains against feeding, thus resulting in lower weight losses and numbers of damaged seeds compared with untreated grains [206]. When Ocimum suave as dry or ground leaves and essential oil was applied to $S$. cerealella in maize and sorghum, all treatments evoked higher mortalities in the moths, as well as significant reductions in the progeny produced by the insects [207]. Furthermore, individual plant secondary metabolites exhibit significant biological properties affecting S. cerealella. For instance, when two sesquiterpene lactones, isolated from the surface of leaves and flowers of Cyrtocymura cincta (Griseb.), were tested at 250 and $500 \mathrm{ppm}$ in the diet of $S$. cerealella they lowered the percentage of adult emergence and produced malformations in adults, altered the oviposition capacity and viability of eggs laid and ceased the production of viable offspring [183]. In another study, the fumigant activity of A. sativum essential oil and its two major components, diallyl disulfide and diallyl trisulfide, expressed as a $50 \%$ lethal concentration for the adult moths, was calculated at $1.33,0.99$ and $1.02 \mu \mathrm{L} / \mathrm{L}$ air space, respectively. Additionally, behavioral deterrent activities were noticed, along with reduced adult longevity and inhibited oviposition by more than $70 \%$ at a concentration of $1.5 \mu \mathrm{L} / 25 \mathrm{~g}$ [143]. Considering the mode of action of diallyl trisulfide treatment, it was found to provoke a decrease in the cuticular chitin content of S. cerealella and to reduce the thermal stability and crystallinity of chitin [144]. Diallyl trisulfide was also found to accelerate the rate of metabolism in males at $\mathrm{LC}_{10}$, leading to the accumulation of greater levels of total soluble sugar to support life activities and to the increased synthesis of proteins to resist an adverse environment [145]. Furthermore, female circadian mating rhythms and calling periodicity changed significantly after diallyl trisulfide treatment, whereas mating frequency and mating duration declined [146]. A recent study proved the ovicidal effect of extracts of Tithonia diversifolia (Asteraceae) flowers and Psychotria prunifolia (Rubiaceae) leaves, as well as astilbin from Dimorphandra mollis (Fabaceae) flowers if applied at $1 \%(\mathrm{w} / \mathrm{w}$ or $\mathrm{m} / \mathrm{m})$ to eggs of S. cerealella, on grains of Triticum aestivum (Poaceae) and on the surfaces of Petri dishes [184]. When Adeyemo and co-authors applied the powders and extracts of Framomum melegueta on paddies at 0.1 to $0.8 \mathrm{~g}$ and $1 \%$ to $5 \%$, respectively, they observed the significant reduction or prevention of S. cerealella adult emergence and an increase in the developmental period as well as the reduction or prevention of paddy seed weight loss [140]. In another study, the petroleum ether extract of $A$. calamus at the application rates of 1000,500 and $250 \mu \mathrm{g} / \mathrm{g}$ and the acetone extract at 1000 and $500 \mu \mathrm{g} / \mathrm{g}$ completely inhibited the emergence of $\mathrm{S}$. cerealella adults [139]. In a fumigant bioassay the essential oil of Coriandrum sativum was tested against $S$. cerealella and the $\mathrm{LD}_{50}$ was calculated at $18.76 \mu \mathrm{g} / \mathrm{cm}^{3}$. Camphor was considered the most active ingredient, with an $\mathrm{LD}_{50}$ of $19.31 \mu \mathrm{g} / \mathrm{cm}^{3}$ [177]. Furthermore, Nazeri and co-authors studied the fumigant toxicity and sublethal effects of essential oils from Artemisia khorassanica Podl. and Artemisia sieberi Besser on adults of S. cerealella. They found that $A$. khorassanica $\left(\mathrm{LC}_{50}=7.38 \mu \mathrm{L} /\right.$ liter air) was a more active fumigant than $A$. sieberi $\left(\mathrm{LC}_{50}=9.26\right.$ $\mu \mathrm{L} /$ liter air), and that the insecticidal effect of $A$. khorassanica $\left(\mathrm{LT}_{50}: 9.01 \mathrm{~h}\right)$ was faster than that of $A$. sieberi $\left(\mathrm{LT}_{50}=14.37 \mathrm{~h}\right)$. Lastly, the fecundity of $S$. cerealella was reduced by $25.29 \%$ and $35.78 \%$ following exposure to sublethal concentrations of A. sieberi and A. khorassanica, 
respectively [157]. The essential oil of Clausena anisata completely inhibited the viability of the larvae and the emergence of adult butterflies from the dose of $0.5 \mu \mathrm{L} / \mathrm{mL}$, contrary to Polyalthia longifolia EO, which respectively recorded $10.0 \%$ and $50.0 \%$ at the dose of $3 \mu \mathrm{L} / \mathrm{mL}$ [174]. The leaf essential oils from Cupressus lusitanica and Eucalyptus saligna were proven to exhibit contact toxicity and air-in-space fumigation against $S$. cerealella, with $\mathrm{LC}_{50}$ values of $0.11 \% \mathrm{v} / \mathrm{w}$ and $7.02 \mu \mathrm{L} / \mathrm{L}$, respectively [181]. In another study, Neroli bigarad oil $\left(1.70 \mu \mathrm{g} / \mathrm{cm}^{3}\right)$ was the most toxic against $S$. cerealella, followed by Citrus aurantium $\left(1.80 \mu \mathrm{g} / \mathrm{cm}^{3}\right)$ and Artemisia vulgaris $\left(1.81 \mu \mathrm{g} / \mathrm{cm}^{3}\right)[58,170]$.

The Indian meal moth (Plodia interpunctella Hubner Pyralidae) is an economically important pest affecting various food products, including cereals, grains and various dry food products. For the most part, the references on plant extracts used to combat $P$. interpunctella report on essential oils. In this context, when the fumigant toxicity of the C. copticum EO was assessed against growth stages of P. interpunctella, it was concluded that the adults were about 500 times $\left(\mathrm{LC}_{50}=257.83 \mu \mathrm{L} / \mathrm{m}^{3}\right.$ air and $\mathrm{LC}_{90}=598.94 \mu \mathrm{L} / \mathrm{m}^{3}$ air) more susceptible than other growth stages. Furthermore, last-instar larvae ( $\mathrm{LC}_{50}$ $=91.36 \mu \mathrm{L} / \mathrm{L}$ air and $\mathrm{LC}_{90}=213.79 \mu \mathrm{L} / \mathrm{L}$ air $)$ and pupae $\left(\mathrm{LC}_{50}=105.69 \mu \mathrm{L} / \mathrm{L}\right.$ air and $\mathrm{LC}_{90}=203.24 \mu \mathrm{L} / \mathrm{L}$ air $)$ were significantly more susceptible than eggs $\left(\mathrm{LC}_{50}=184.61\right.$ $\mu \mathrm{L} / \mathrm{L}$ air and $\mathrm{LC}_{90}=435.32 \mu \mathrm{L} / \mathrm{L}$ air) [226]. In a choice test of P. interpunctella using an olfactometer, the strongest repellency was exhibited by the essential oil of $A$. graveolens $(100 \%)$, Thymus vulgaris (100\%) and R. officinalis L. (93.33\%) and the weakest repellency by Hyossopus officinalis (7.69\%) and P. sativum (9.48\%) [152]. In another study, the essential oils of myrtle, laurel, marjoram and lemon provoked mortality at $41.66 \%, 50.83 \%, 57.50 \%$ and $26.66 \%$ for $P$. interpunctella eggs, respectively. At the moderate dose $(50 \mu \mathrm{L} / \mathrm{L}$ air $)$ the $\mathrm{LT}_{99}$ values of the most effective essential oil (savory) was $81.88 \mathrm{~h}$ for the eggs of the moth [205]. The eggs of $P$. interpunctella were the most tolerant to the essential oils of garlic, birch (Betula lenta), cinnamon (C. zeylanicum) and aniseed (Pimpinella anisum), with $\mathrm{LC}_{90}$ values ranging from 22.02 to $72.42 \mu \mathrm{L} / \mathrm{L}$ air [147]. The essential oils of oregano and savory were highly effective against $P$. interpunctella, with $100 \%$ mortality obtained after $24 \mathrm{~h}$ at $9 \mu \mathrm{L} / \mathrm{L}$ air [205]. The essential oil of Armoracia rusticana was assessed against $P$. interpunctella and adults were found to be much more susceptible than pupae; its relatively low fumigant effect on pupae might be due to the gas vapor being unable to permeate through the thick wall of the pupal case [54]. After $9 \mathrm{~h}$ of exposure, the $\mathrm{LC}_{50}$ values of the essential oil from S. hortensis and Z. officinale for P. interpunctella were $139.8 \mu \mathrm{L} / \mathrm{L}$ and $69.05 \mu \mathrm{L} / \mathrm{L}$ air, respectively $[129,218]$. In another study, insect-resistant films and anti-insect polymer strips containing cinnamon oil were developed to protect food products from the Indian meal moth $[168,169]$. When Thymus daenensis essential oil was tested for its fumigant toxicity against first- and third-instar larvae and adults the $\mathrm{LC}_{50}$ values were calculated at $25.32,34.80$ and $0.27 \mu \mathrm{L} / \mathrm{L}$, respectively [217]. The fumigant activity of essential oil vapors distilled from sweet basil $O$. basilicum and spearmint Mentha spicata were tested against $P$. interpunctella at 0.5 to $1.500 \mu \mathrm{L} / \mathrm{L}$ air. Adult moths were the most sensitive, with a notable mortality $(>80 \%)$ recorded after exposure to low doses such as $2.5 \mu \mathrm{L} / \mathrm{L}$, but other than that, basil and spearmint oils did not show satisfactory overall insecticidal activity against the moth [202]. In another study, beads of encapsulated coriander and basil EO proved to be efficacious in funnel traps in stores of almonds and pet foods against $P$. interpunctella [178]. When two Indian spices, namely, Trachyspermum ammi and $M$. fragrans, were studied for the fumigant activity of their essential oil at $10 \mu \mathrm{L} / \mathrm{L}$ air against $P$. interpunctella, T. ammi was found to be comparatively more effective [204]. Similarly, the lethal and sublethal effects of essential oils of $A$. khorassanica and Vitex pseudo-negundo were studied on P. interpunctella. The fumigant toxicity of $A$. khorassanica $\left(\mathrm{LC}_{50}=9.60 \mu \mathrm{L} / \mathrm{L}\right.$ air) was higher than that of $V$. pseudo-negundo $\left(\mathrm{LC}_{50}=23.05 \mu \mathrm{L} / \mathrm{L}\right.$ air $)$ and the exposure to sublethal concentrations of A. khorassanica negatively affected the protein, lipid and glycogen contents of the larvae coming from treated adults [158]. In another study, the toxicity of the essential oils isolated from parsley, P. crispum and coriander, C. sativum, was studied, and the $\mathrm{LC}_{50}$ values were calculated to be 55.197 and $50.956 \mu / L$ air for P. interpunctella larvae, respectively. In another 
study, M. piperita and S. officinalis were incorporated into polylactic acid solution to test for contact toxicity on P. interpunctella. The product showed higher contact toxicity than the pure essential oil because the ploylactic acid nanofibers cause surface tension and longer efficiency times due to the slow release. Moreover, M. piperita showed higher toxicity than S. officinalis [165]. A multilayered insect-proof film preventing contamination with $P$. interpunctella was developed based on garlic and onion EOs and their compounds chosen as efficient anti-insect agents [142]. When lavender (L. angustifolia), peppermint (M. piperita), geranium (Geranium maculatum), palmarosa (Cymbopogon martini (Roxb.) Wats), eucalyptus (E. globulus) and bergamot (Citrus bergamia Risso) were used against adult moths, the contact toxicity assay showed that the $\mathrm{EO}$ from palmarosa was the most toxic, with an $\mathrm{LD}_{50}$ value of $22.8 \mu \mathrm{g} / \mathrm{cm}^{2}$, and the greatest fumigant toxicity was found with the EO from eucalyptus, with a $\mathrm{KT}_{50}$ value of $8.34 \mathrm{~min}$ [171]. In another study, low-density polyethylene-laminated polypropylene films printed with ink incorporating microencapsulated cinnamon oil using a large-scale film production system effectively repelled Indian meal moth larvae [167]. Eucalyptus dives oil and constituent 3-carvomenthenone, cyclohexanone ( $\mathrm{LD}_{50}$ against, 2.45 and $\left.3.63 \mu \mathrm{g} / \mathrm{cm}^{3}\right)$, methylcyclohexanone $\left(2.95\right.$ and $\left.4.24 \mu \mathrm{g} / \mathrm{cm}^{3}\right)$ and seudenone (3.02 and $4.44 \mu \mathrm{g} / \mathrm{cm}^{3}$ ) were proven to have fumigant activity against larvae and adults of Plodia interpunctella [190]. In a recent study, geranium EO (G. maculatum) was used to develop micro and nanoemulsions, adding Tween 80 as surfactant which was stable at $25{ }^{\circ} \mathrm{C}$ for 60 days. This formulation can increase the insecticidal efficacy of EO twofold [192]. The essential oil distilled from Lippia turbinata ("poleo") was insecticidal on P. interpunctella larvae [198]. In a recent study, the essential oils (O. vulgare, P. anisum and Tanacetum cinerariifolium) and four plant extracts (Agastache rugosa, Capsicum annuum, C. reticulata and Ginkgo biloba) were proven to be repellent against $P$. interpunctella. Additionally, O. vulgare and T. cinerariifolium had greatest repellent efficacy against the moth larvae [46]. In another study, insect-resistant adhesives were developed for application to a cardboard packaging system for preventing P. interpunctella larvae infestation. Cinnamon EO was used as an, encapsulated with maltodextrin, $\beta$-cyclodextrin and polyvinyl alcohol, in corn starch paste that was able to control insect penetration in the distribution and storage steps [166].

There are fewer studies referring to other essential oil plant extracts and powders used for controlling P. interpunctella. In a study conducted by Akkineye and co-authors the root bark powder of Cleisthopholis patens at 1.0, 2.0 and $3.0 \mathrm{~g} / 20 \mathrm{~g}$ of the maize evoked $100 \%$ adult moth mortality within $72 \mathrm{~h}$ of application, whereas the stem bark powder of $C$. patens at $1.0,2.0$ and $3.0 \mathrm{~g} / 20 \mathrm{~g}$ of the maize produced $78 \%-100 \%$ moth mortality within $72 \mathrm{~h}$ of treatment. The leaf powder was moderately effective against the adult moth at $3.0 \mathrm{~g} / 20$ g of the maize grain, evoking $70 \%-80 \%$ adult mortality within $96 \mathrm{~h}$ of treatment [175]. The methanol extracts of Peganum harmala, Ajuga iva, R. officinalis L., L. stoechas, Lavandula dentata, Cistus ladanifer, Cistus salviaefolius, Cistus monspeliensis, Centaurium erythraea and Launaea arborescens were tested at $500 \mathrm{ppm}$ on post-embryonic development parameters of P. interpunctella. Most plant extracts provoked a notable decrease in larval weight, causing significant alterations on pupation and adult emergence. When applied at 500, 750 and $1000 \mathrm{ppm}$, they also affected physiological parameters such as larval reserve substances and the midgut activities of hydrolytic and detoxification enzymes [141]. Plant protease inhibitors regulate proteolytic processes in insects and are thus considered to be a potential safe weapon against insect pests, either through their direct application or via their expression in transgenic plants. In this regard, medicinal legume plants, namely, senna (Cassia angustifolia) and fenugreek (T. foenum-graecum), could be used for their total proteolytic inhibitory activity against the Indian meal moth larval midgut [164]. Another study investigated a bioinspired cyanogenic grain coating with amygdalin as a cyanogenic precursor, mimicking the feeding-triggered release of hydrogen cyanide found, for example, in bitter almonds. Upon feeding of coated cyanogenic wheat grains to T. molitor L., R. dominica Steph. (lesser grain borer) and P. interpunctella, their reproduction, as well as consumption rate, were significantly reduced, whereas their germination ability increased compared to non-coated grains [216]. Diterpene resin acids are important components of oleoresin 
and greatly contribute to the defense strategies of conifers against herbivorous insects and also function as insect juvenile hormone antagonists that interfere with the juvenile hormone-mediated binding of the $\mathrm{JH}$ receptor methoprene-tolerant and steroid receptor coactivator protein [211]. Hexane extracts of the roots of Piper sarmentosum Roxb. and constituent asaricin 1, isoasarone 2 and trans-asarone 3 were toxic to P. interpunctella [212]. Plant juvenile hormone disruptor activity is concentrated in certain plant groups, families and their plant metabolites, which have insect group-specific activity. Reciprocal diversification has occurred between plants and insects through the evolution of secondary plant metabolism and juvenile hormone receptors, respectively, and that plant metabolites could be developed into insect group-specific pesticides with limited effects on non-target species [227].

The rice moth (Corcyra cephalonica Stainton, Pyralidae) feeds on both seeds and flour. Most of the literature on natural substances against C. cephalonica investigate essential oils, although constraints including a lack of data for single or multiple components of essential oils in terms of their sorption, tainting and residues in food commodities, and registration protocols are yet to be highlighted [10]. M. piperita and Piper nigrum EO LC 50 values against the larvae were calculated at 343.9 and $530.5 \mu \mathrm{L} / \mathrm{L}$ of air $72 \mathrm{~h}$ after commencement [113]. In a fumigation assay, coriander and eucalyptus oils at $130 \mu \mathrm{g} / \mathrm{cm}^{2}$, caused $100 \%$ toxicity to C. cephalonica within $24 \mathrm{~h}$ of treatment, whereas pine oil revealed $90 \%$ mortality at the same concentration after $72 \mathrm{~h}$ of treatment. In a contact assay, the test oils were effective against adults producing about $90 \%$ toxicity only after $72 \mathrm{~h}$ of treatment [82]. The fumigant toxicity of Origanum majorana essential oil against C. cephalonica adults and larvae was calculated, with $\mathrm{LC}_{50}$ values of 11.31 and $49.83 \mu \mathrm{L} / \mathrm{L}$ air, respectively. The contact toxicity against adult, pupa, larvae and eggs was observed, with $\mathrm{LC}_{50}$ values of $2.54,0.95,2.78$ and $0.49 \mu \mathrm{L} / \mathrm{L}$, respectively. The acetylesterase inhibition activity of $O$. majorana $\mathrm{EO}$ was observed against adults and larvae, with $\mathrm{LC}_{50}$ values of 35.89 and $118.54 \mu \mathrm{L} / \mathrm{mL}$, respectively [208].

Plant extracts of Lawsonia inermis, petroleum ether (pet ether) extract, produced a complete inhibition of the moths, and were thus recorded as the best repellants (96.70\%), whereas Parthenium hysterophorus, Dalbergia sissoo (acetone extracts) and Acacia nilotica (acetone and pet ether extracts) showed 70\% repellency. However, a minimum of 30.81\% repellency was exhibited by Eucalyptus rudis (pet ether extract) [138]. Larvicidal and pupicidal effects of Dryopteris filix-mas root and rhizome ethanolic extracts were studied against the third-instar larvae of C. cephalonica. D. filix-mas extract $0.20 \%$ (v/w) caused $100 \%$ larval mortality. The plant extracts reduced the pupation percentage, pupal death and adult emergence, indicating absolute toxicity to the pest [185]. Essential oils isolated from pine (Pinus longifolia), eucalyptus (Eucalyptus obliqua) and coriander (C. sativum) were screened for contact and fumigant activities. In a fumigation assay, coriander and eucalyptus oils at $130 \mu \mathrm{g} / \mathrm{cm}^{2}$ caused $100 \%$ toxicity within $24 \mathrm{~h}$ of treatment, whereas pine oil revealed $90 \%$ mortality at the same concentration after $72 \mathrm{~h}$ of treatment. In a contact assay, the test oils were effective against adults, producing about $90 \%$ toxicity only after $72 \mathrm{~h}$ of treatment [82]. High larvicidal, adulticidal, antifeedant and oviposition deterrent activities were exhibited by hexane extracts of Glossocardia bosvallia 30 and $60 \mathrm{mg} / \mathrm{mL}$ in wheat grains [193]. Acetone extracts of young leaves, old leaves, flowers and stems of groundnut plant with Trichogramma chilonis (Ishii) and C. zastrowi sillemi (Esben-Petersen) revealed their kairomonal activities under in vitro conditions against C. cephalonica [154].

Fumigation with essential oils of Eucalyptus platyphylla and M. piperita, at different concentrations, on adults of the almond moth, Ephestia cautella Walker (Pyralidae), showed that those of M. piperita caused the highest mortality rates [191]. The almond moth, E. cautella Walker, comes from the family Pyralidae. In a filter paper contact toxicity bioassay, potent toxicity was produced from buchu leaf, niaouli and rosemary oils at $2.4 \mathrm{mg} / \mathrm{cm}$ and armoise, cypress, galbanum and mace oils at $4.7 \mathrm{mg} / \mathrm{cm}$ against E. cautella Walker. In vapor phase toxicity bioassays with larvae, cypress, galbanum, niaouli and rosemary oils were much more effective in closed containers than in open containers, indicating that the lethal effects of these oils were largely because of action in the vapor phase [159]. 
In another study, early eggs of E. cautella Walker showed a lower $\mathrm{LC}_{50}$ value of 48.56 $\mu \mathrm{L} / \mathrm{L}$, compared to $77.75 \mu \mathrm{L} / \mathrm{L}$ with late eggs, when the essential oil of Lantana camara was used to treat them with fumigation [194]. Finally, in a recent study it was found that extracts of Calotripis procera roots displayed the most potent activity, with $50 \%$ of E. cautella Walker eggs not hatching at $10.000 \mathrm{ppm}(1 \%)$. The chemical composition analysis of the extracts demonstrated a high presence of cardenolides, including calactin, uscharidin, $15 \beta$-hydroxy-calactin, $16 \beta$-hydroxy-calactin and 12 $\beta$-hydroxy-calactin [162]. When five Eucalyptus species, namely, E. camaldulensis, E. astringens, E. leucoxylon, E. lehmannii and E. rudis, were assessed for their fumigant activity on Ephestia spp., the E. camaldulensis essential oil was more toxic against E. cautella and Ephestia kuehniella Zeller and the $\mathrm{LC}_{50}$ values were 11.07 and $26.73 \mu \mathrm{L} / \mathrm{L}$ air, respectively. In all cases the fumigant activity was strongest for the summer season oils and E. cautella was the most sensitive species [189]. In a choice test, rosemary and eucalyptus revealed high percentages of repellency after 5 days, at $72 \%$ and $50 \%$, respectively, in the case of E. kuehniella and $71 \%$ and $54 \%$, respectively, in case of E. cautella.

The most effective oil in enhancing the potency of an entomopathogenic fungus, Metarhisium anisopliae, against insect species was rosemary and it decreased the $\mathrm{LC}_{50}$ for E. cautella and E. kuehniella, respectively [148]. When the oils of Capsicum frutescens, Anacardium occidentale, Monodora tenuifolia, Xylopia aethiopica and Ricinus communis were studied at $0.5 \mathrm{~mL}$ and $1.0 \mathrm{~mL}$ dosages against the egg, larvae and adults of E. cautella, A. occidentale was found to be the most effective [150]. E. angustifolia ethyl acetate and aqueous extracts caused mortality of E. cautella, observed after 24 and $48 \mathrm{~h}$ of treatment [186]. The ethanolic oil extract of M. tennuifolia achieved $100 \%$ E. cautella moth mortality and its effect was significantly $(p<0.05)$ different from that of all other extracts. However, petroleum ether increased the effect of the other extracts on the survival of the moth, as reflected by their $\mathrm{LD}_{50}$ values. Regardless of the solvent used, all the oils reduced the hatchability of the eggs of the insect, and the adult emergence of the insect was prevented by all the oil extracts [151]. Another study was conducted to evaluate the activity of the neem oil of Azadirachta indica and Bacillus thuringiensis alone and in combination in controlling E. cautella. The B. thuringiensis $\mathrm{LC}_{50}$ was at $0.1 \%$ concentration after one day of treatment, whereas the effect of the combination of $B$. thuringiensis and neem oil showed an increase in the mortality rate of larvae treated with the combination, as compared with the treatment with each separately [160].

The Mediterranean flour moth (also known as the mill moth, Ephestia kuehniella) is another species from the same family and genus as E. cautella, and is similarly an important pest affecting grains and flour. E. camaldulensis essential oil was toxic against E. cautella and the $\mathrm{LC}_{50}$ value was $11.07 \mu \mathrm{L} / \mathrm{L}$ air, whereas the median lethal time $\left(\mathrm{LT}_{50}\right)$ value was $13.49 \mathrm{~h}$ [189]. Exposure to vapors of essential oils from anise and cumin resulted in $100 \%$ mortality of E. kuehniella eggs. Oregano achieved mortality as high as $89 \%$, whereas eucalyptus and rosemary achieved $45 \%$ and $65 \%$, respectively [180]. In another study, the average mortality rates with a dose of $10 \mu \mathrm{L}$ of essential oils of Origanum acutidens, Satureje hortensis, Hypericum scabrum, T. vulgaris, Micromere fruticosa, Salvia limbata C. A. Meyer, S. nemerosa and Hyssoppus officinalis were approximately $74 \%, 66 \%, 73 \%, 4 \%, 12 \%$, $7 \%, 10 \%$ and $14 \%$ for S. granarius, and $79 \%, 62 \%, 72 \%, 24 \%, 24 \%, 6 \%, 0 \%$ and $14 \%$ for $E$. kuehniella, respectively [117]. Essential oil vapors of Micromeria fruticosa, Nepata racemosa and $O$. vulgare tested at $8 \mu \mathrm{L} / \mathrm{L}$ air provoked $100 \%$ mortality of the larvae (third instar) of E. kuehniella after $120 \mathrm{~h}$ of treatment [203]. Essential oil vapors from the plant species O. acutidens achieved $100 \%$ mortality with a dose of $2 \mu \mathrm{L} / \mathrm{L}$ air within $96 \mathrm{~h}$ in third-instar larvae of E. kuehniella [124]. Assessing the ovicidal activity of five essential oil vapors distilled from savory Satureja thymbra, laurel L. nobilis, myrtle Myrtus communis, lemon Citrus limon and marjoram O. majorana, the essential oil obtained from savory produced $100 \%$ mortality for the eggs of E. kuehniella and at the moderate dose $(50 \mu \mathrm{L} / \mathrm{L}$ air) the $\mathrm{LT}_{99}$ values of the most effective essential oil, savory, were 158.50 and $81.88 \mathrm{~h}$ for the eggs of E. kuehniella [172]. The effects of vapors of essential oils from garlic, birch (B. 
lenta), cinnamon (C. zeylanicum) and aniseed (Pimpinella anisum) were studied on eggs of E. kuehniella. Generally, garlic and birch essential oils were more toxic to the eggs of the tested insect species than cinnamon and aniseed essential oils. Garlic and birch essential oils were found to be very promising since they also showed high fumigant toxicity on eggs of $E$. kuehniella [147]. When the essential oils from oregano, Origanum onites; savory, Satureja thymbra; and myrtle, Myrtus communis were tested on E. kuehniella adults, oregano and savory achieved 100\% mortality, obtained after $24 \mathrm{~h}$ at $25 \mu \mathrm{L} / \mathrm{L}$ air [205]. Pistacia lentiscus oil was toxic to E. kuehniella with $\mathrm{LC}_{50}=1.84 \mu \mathrm{L} / \mathrm{L}$ and $\mathrm{LC}_{95}=5.14 \mu \mathrm{L} / \mathrm{L}$, respectively. At $136 \mu \mathrm{L} / \mathrm{L}$ air, fecundities and hatching rates were 78 eggs/female and 29.49\% [213]. The preventative properties of Cinnamomum camphora and Syzygium aromaticum to the E. kuehniella 5th-instar larvae penetration to packaged cereals were tested by Allahvaisi and co-workers. Four days after the initiation of the experiment, the results showed that S. aromaticum had more of a repellency effect than C. camphora [165]. In another study, the toxicity of $S$. hortensis essential oil was investigated against 12-to-14-day-old larvae of the Mediterranean flour moth. $\mathrm{LC}_{50}$ values were calculated as $80.9 \mu \mathrm{L} / \mathrm{L}$ after $9 \mathrm{~h}$ for $E$. kuhniella, and additional repellency was evident [129]. Origanum majorana and Citrus limon were the most effective essential oils against $E$. kuehniella and the $\mathrm{LC}_{50}$ and LC 99 values were estimated to be 3.27 and $5.13 \mu \mathrm{L} / \mathrm{L}$ of air for marjoram and 4.05 and $5.57 \mu / \mathrm{L}$ of air for lemon essential oils at the longest exposure time [173]. O. basilicum and Z. officinale oils produced $100 \%$ mortality within $24 \mathrm{~h}$ with doses of $32 \mu \mathrm{L} / \mathrm{L}$ air for E. kuehniella adults, whereas $A$. graveolens oil was the most active against E. kuehniella larvae. The oils were selected as having a possible application as a $30 \%$ aqueous solution for the protection of wheat flour [153]. In another study, the insecticidal activity of the essential oil from cardamom, Elettaria cardamomum, on adults of E. kuehniella was investigated, and the highest mortality of these insects was seen after $12 \mathrm{~h}$ [187]. The fumigant toxicity varies with season, essential oil concentration and exposure time, as shown for five Eucalyptus species, as tested by Ben Jemâa and co-authors against E. kuehniella [189]. In another study, the $\mathrm{LC}_{50}$ value for E. kuehniella after $9 \mathrm{~h}$ was calculated as $258.95 \mu \mathrm{L} / \mathrm{L}$ air. In a contact toxicity assay, Z. officinale essential oil's LC $_{50}$ value for E. kuehniella was determined to be $0.61 \mu \mathrm{L} / \mathrm{cm}^{2}$ and its repellency was significant [218]. The toxicity of vapors of the essential oil of Ferula gummosa, Elettaria cardamomum and S. officinalis was tested on the adults and larvae of E. kuehniella and the results indicated that the effect of the essential oil of F. gummosa was the strongest [188]. The insecticidal effect of Cupressus sempervirens horizontalis resin essential oil exposure ( $48 \mathrm{~h}$ ) on E. kuehniella eggs showed $20.83 \%$ mortality at a $100 \mu \mathrm{L} / \mathrm{L}$ air concentration [182]. In another study, the repellent effects of essential oils from L. nobilis and M. communis against adults of E. kuehniella were studied. Around $20.4 \%$ and $10.6 \%$ repellency rates were observed at the lowest concentration $(0.50 \mu \mathrm{L} / \mathrm{L})$ of L. nobilis and Myrtus commonis, respectively. Furthermore, at the highest-concentration $(2.00 \mu \mathrm{L} / \mathrm{L}$ air) repellency rates reached $84.2 \%$, obtained by $L$. nobilis, and $61.3 \%$, obtained by M. commonis [195]. Hematological observations showed that in comparison to fumigation, the topical application of Callistemon viminalis and Ferula gummosa oils caused a drastic reduction in the total hemocyte counts of treated larvae in a dose-dependent manner at all time intervals [161]. When T. daenensis essential oil and its toxicity was investigated against the first- and third-instar larvae and adults of E. kuehniella in terms of fumigant toxicity, the adult stage was more sensitive, whereas the sensitivity of larvae decreased from the first to the third instar [217]. Essential oils of the resins of Pinus brutia and P. pinea were found to be insecticidal on E. kuehniella eggs [182]. When essential oils from sweet basil $O$. basilicum and spearmint Mentha spicata L. were tested against on E. kuehniella no significant differences were found in insecticidal action and both oils produced a notable level of mortality (>80\%) after exposure to low doses, such as $2.5 \mu \mathrm{L} /$ liter. Larvae and pupae were the most tolerant stages in all cases [202]. In another study, the fumigant toxicity potential of L. angustifolia oil ( $\mathrm{LC}_{50}=19 \mu \mathrm{L} / \mathrm{L}$ air) was greater than that of $R$. officinalis oil $\left(\mathrm{LC}_{50}=\right.$ $28 \mu \mathrm{L} / \mathrm{L}$ air) as regards E. kuehniella larvae [196]. Essential oils of Artemisia haussknechtii and Citrus vulgaris exhibited LC $_{50}$ values of 479.13 and $110.98 \mu \mathrm{L} / \mathrm{L}$ air E. kuehniella [155]. 
The $\mathrm{LC}_{50}$ value of Ziziphora clinopodioides Lam. oil was estimated to be $54.61 \mu \mathrm{L} / \mathrm{L}$ air for E. kuehniella larvae and $1.39 \mu \mathrm{L} / \mathrm{L}$ air for adults [219]. Beads of encapsulated coriander and basil EO were tested in funnel traps in stores of almonds and pet foods for 2 months. The number of adult moth E. kuehniella dead captures was similar with either coriander or basil EO beads and with vapona tablets, although there were more insects alive in the control [178]. The $\mathrm{LC}_{50}$ values of C. sativum and P. crispum essential oils were 62.633 and $52.412 \mu \mathrm{L} / \mathrm{L}$ air against E. kuehniella [179]. Purslane oil (bulk) exhibited mortality against larvae of E. kuehniella, with $66.64 \%, 55.21 \%, 45.32 \%$ and $18.61 \%$ mortality rates gained at 3\%, $1.5 \%, 0.5 \%$ and $0.2 \%$ concentrations, respectively. However, the mortality values amounted to $96.64 \%, 88.68 \%, 78.79 \%$ and $60.61 \%$ when the larvae were exposed to $0.1 \%, 0.5 \%, 0.05 \%$ and $0.005 \%$ concentrations of nano-purslane, respectively [215]. Concentrations of 5.42 and $6.81 \mu \mathrm{L} / \mathrm{ml}$ were obtained as $\mathrm{LC}_{50}$ values for C. copticum essential oils and thymol, and these significantly influenced the enzymatic activities of E. kuehniella [163]. As for E. cautella, as well as for E. kuehniella, the potential activities of four essential oils (anise, eucalyptus, garlic and rosemary) and the microbial agent $M$. anisopliae and their combinations were evaluated. The most effective oil in enhancing the potency of $M$. anisopliae was rosemary, against both insect species [149]. The $\mathrm{LC}_{50}$ value for $M$. longifolia essential oil in regard to E. kuehniella was 21,352 ppm, whereas this value for the nanoemulsion was 14,068 ppm. In addition, M. longifolia oil had lower durability and the $50 \%$ persistent time $\left(\mathrm{PT}_{50}\right)$ was 2.39 days, compared to the nanoemulsion's value of $\mathrm{PT}_{50}=17.13$ days, in the highest concentration of essential oil [199]. In another study, the effect of the essential oil of Origanum vulgaris was tested on the reproduction and mortality of the flour moth $E$. kuehniella. It was found to affect the pupal development. It also disrupted the reproduction of exuviated adults by extending the preoviposition period and reducing the period of egg-laying and fecundity because fecundated females could not live more than four days compared to the control group [209]. In a recent study, wormwood (A. herba-alba), O. vulgare and rue (Ruta montana) were evaluated for their repellent and fumigant toxic potential against larvae of the flour moth E. kuehniella. Origanum oil was the most repellent, with a $67 \%$ repellency rate, followed by Artemisia oil (46\%) at $120 \mu \mathrm{L} / \mathrm{mL}$ after $2 \mathrm{~h}$ of exposure. The oil of $R$. montana showed attractant activity against the larvae and was the most toxic, with $56.7 \%$ of larval mortality in the first $24 \mathrm{~h}$. The median lethal concentrations $\left(\mathrm{LC}_{50}\right)$ recorded were 11.6, 175.4 and $1100.0 \mu \mathrm{L} / \mathrm{L}$ air for the plant oils $R$. montana, O. vulgare and $A$. herba-alba, respectively. R. montana and $O$. vulgare essential oils were shown to be efficient, with high toxic and repellent properties against E. kuehniella larvae [156]. The lethal concentration values of $O$. basilicum, Mentha pulegium and Ruta graveolens were, respectively, $0.96,0.3$ and $1.02 \mu \mathrm{L} / \mathrm{L}$ air on E. kuehniella [201]. In a recent study, highly synergistic effects, considering antifeedance, relative growth rate and relative consumption rate, were observed in 1.14 and $1.7 \mu \mathrm{L} / \mathrm{L}$ air of L. angustifolia in combination with $200 \mathrm{~Gy}$ of gamma radiation [197].

\section{Practical Concerns}

In recent years, environmental contamination, pesticide resistance and the destruction of nontarget organisms have led to increased support for environmentally safe pesticide alternatives to the use of synthetics. The research on botanical pesticides for pest management practices has intensified because they demonstrate a wide range of bioactivities and display contact and fumigant toxicity, as well as repellent activity, effects on oviposition and feeding deterrence. Additionally, many botanicals display low mammalian toxicity and rapid degradation. In this regard, EOs may prove to be reasonable alternatives to the more persistent synthetic pesticides as tools to control stored-product insects, even combined with gamma radiation or diatoms [228]. However, the number of commercial biopesticides based on EOs remains low and the key challenges include the stabilization processes (e.g., microencapsulation) and authorization requirements, as well as plant growing conditions and extraction processes [229]. Loading EOs in nanostructured systems represents a potential solution to their sensitivity to $\mathrm{pH}$, oxygen, light and moderate temperatures. The 
use of biopolymers as nanocarriers provides the controlled release of the active ingredient, also ameliorating the issues of biodegradability, biocompatibility, environmental safety, low toxicity and competitive production costs [230]. Other than EO plant-based products (powders, extracts, essential oils, etc.) are also cost-effective alternatives to synthetic insecticides, and these are of great importance regarding insect pest control strategies for stored food commodities. To date, there have been serious bottlenecks in regard to their commercialization and standardization, including their low priority in agricultural policy, as well as biosafety and intellectual property rights issues [231]. The commercialization of new botanical insecticides and the market expansion of existing botanicals has lagged considerably, considering the academic interest demonstrated in the past 20 years. Some countries (such as Turkey, Uruguay, the United Arab Emirates and Australia) have relaxed regulatory requirements for specific plant extracts and oils, whereas in North America and the European Union, stricter requirements have slowed progress toward the commercialization of new products. Thus, botanicals are likely to remain niche products for some countries, while having the greatest impact in places where the source plants are readily available and where conventional products are both expensive and dangerous to users [9].

\section{Conclusions}

Plant extracts have been tested mainly in the form of essential oils against stored grain pests and, in some cases, the research has extended as far as including the main components of these oils.

Regarding mites, studies have addressed the effects on pests, as well as predatory species. There are three substances that have shown the best results when tested in the lowest of concentrations, even lower than benzyl-benzoate (an already industrially available product). These substances are components of the clove bud (E. caryophyllata) essential oil, methyleugenol, forget-me-not (M. arvensis) essential oil and benzaldehyde of P. persica. Furthermore, cinnamon (naturally containing benzyl-benzoate) has been proven to be effective against T. putrescentiae, T. molitor, S. oryzae, R. dominica, P. interpunctella and E. kuhniella as a contact toxicant to larvae or adults, as a repellant or as a fumigant. However, detailed knowledge of the lethal or non-lethal effects of botanical pesticides on beneficial insects is important for integrated pest management (IPM).

Regarding the impact of pesticides on Coleoptera and Lepidoptera, many plant species have been studied for their pesticidal properties. C. copticum was found to be active against T. confusum, S. granarius, P. interpunctella and E. kuehniella, whereas basil was effective as a fumigant for S. zeamais, O. surinamensis and E. kuehniella. Most interestingly various Citrus species were effective as fumigants, contact toxicants and as repellants, for both Coleopterans (T. castaneum, S. oryzae and C. ferrugineus) and Lepidoptera (P. interpunctella, S. cerealella and E. kuehniella). In addition, in regard to Lepidoptera, many substances have been evaluated for sublethal effects (C. cincta lowered the percentage of adult emergence, and $A$. sativum reduced adult longevity and inhibited oviposition), whereas for Coleoptera only a few studies of sublethal effects are available.

T. minuta and T. patula extracts were very effective fumigants against T. castaneum Herbst, whereas Eucalyptus spp. extracts were very effective fumigants against the adults of $R$. dominica, T. castaneum and $O$. surinamensis, but were also very effective against Lepidoptera (E. kuehniella, E. cautella, C. cephalonica and P. interpunctella) as fumigants or repellents. $C$. reticulata and $C$. sinensis essential oils were among the few substances found to be effective against Coleoptera (T. castaneum) larvae, as was the garlic essential oil, causing necrosis to the larvae, pupae and adults of T. molitor $\mathrm{L}$. The fumigant properties of $C$. citratus and $Z$. officinale were the most effective fumigants against the rice weevil. Furthermore, $A$. judaica and $C$. viminals had two of the lowest $\mathrm{LC}_{50}$ values observed in this entire review. L. salicifolia, L. nobilis and E. floribundi were significantly effective repellents, whereas $O$. acutidens and $C$. reticulata were effective fumigants against $L$. serricorne and C. ferrugineus. 
Finally, regarding Lepidoptera, the essential oil of $C$. anisata was found to be completely toxic to the larval stages, and also inhibited the emergence of adults. M. piperita and $S$. officinalis incorporated into polylactic acid solution showed significant contact toxicity on $P$. interpunctella, whereas M. piperita showed higher toxicity than S. officinalis. The essential oils exhibiting contact toxicity showed higher toxic effects when they were loaded on polymeric nanoparticles, as in the case of palmarosa (C. martinii), geranium (G. maculatum), and peppermint (M. piperita) against P. interpunctella. Eucalyptus spp. was the most tested substance in Lepidoptera, achieving $100 \%$ mortality, and it was effective as a fumigant and exhibited contact toxicity. Additionally, purslane essential oil was very effective against E. cautella in terms of fumigant toxicity, oviposition deterrence and persistence against larvae and adults. M. tennuifolia achieved $100 \%$ E. cautella moth mortality and reduced the hatchability of the eggs, whereas adult emergence was prevented, making it a very useful tool against $E$. cautella; as was birch essential oil, which displayed high fumigant toxicity on the eggs of E. kuehniella. Furthermore, A. graveolens oil was found to be the most active against E. kuehniella larvae, the life stage of the Lepidoptera species that causes the most damage. Finally, the essential oil of O. vulgaris was found to affect pupal development and disrupt the reproduction of exuviated adults by extending the preoviposition period and reducing the period of egg-laying and fecundity.

In general, there is a shortage of tools for combatting stored-product pests, as an alternative to the use of synthetics. With this review, we refer to the botanical substances reported in the literature of recent years to exhibit pesticidal properties of potential utility in the management of stored products.

Author Contributions: Conceptualization, P.N., P.M., Z.A. and N.N.; writing-original draft preparation, P.N., P.M., Z.A. and N.N.; writing-review and editing, P.N., P.M., Z.A. and N.N. All authors have read and agreed to the published version of the manuscript.

Funding: This research received no external funding.

Institutional Review Board Statement: Not applicable.

Informed Consent Statement: Not applicable.

Data Availability Statement: Not applicable.

Conflicts of Interest: The authors declare no conflict of interest.

\section{References}

1. Mesterházy, Á.; Oláh, J.; Popp, J. Losses in the Grain Supply Chain: Causes and Solutions. Sustainability 2020, 12, 2342. [CrossRef]

2. Kim, K.-H.; Kabir, E.; Jahan, S.A. Exposure to pesticides and the associated human health effects. Sci. Total Environ. 2017, 575, 525-535. [CrossRef]

3. Sharma, A.; Kumar, V.; Shahzad, B.; Tanveer, M.; Sidhu, G.P.S.; Handa, N.; Kohli, S.K.; Yadav, P.; Bali, A.S.; Parihar, R.D. Worldwide pesticide usage and its impacts on ecosystem. SN Appl. Sci. 2019, 1, 1-16. [CrossRef]

4. Karabelas, A.; Plakas, K.; Solomou, E.; Drossou, V.; Sarigiannis, D. Impact of European legislation on marketed pesticides-A view from the standpoint of health impact assessment studies. Environ. Int. 2009, 35, 1096-1107. [CrossRef]

5. Pimentel, D. Environmental and Economic Costs of the Application of Pesticides Primarily in the United States. Environ. Dev. Sustain. 2005, 7, 229-252. [CrossRef]

6. Bruinsma, J. World Agriculture: Towards 2015/2030: An FAO Perspective; Earthscan: London, UK, 2003.

7. Ministry of Rural Development and Food. Hellenic Republic. Available online: http://www.minagric.gr/index.php/en/farmermenu-2/plantprotection-menu/biocides-menu (accessed on 11 July 2021).

8. Boyer, S.; Zhang, H.; Lempérière, G. A review of control methods and resistance mechanisms in stored-product insects. Bull. Entomol. Res. 2012, 102, 213-229. [CrossRef]

9. Isman, M.B. Botanical insecticides in the twenty-first century—fulfilling their promise? Annu. Rev. Entomol. 2020, 65, 233-249. [CrossRef]

10. Rajendran, S.; Sriranjini, V. Plant products as fumigants for stored-product insect control. J. Stored Prod. Res. 2008, 44, 126-135. [CrossRef]

11. Stejskal, V.; Vendl, T.; Aulicky, R.; Athanassiou, C. Synthetic and Natural Insecticides: Gas, Liquid, Gel and Solid Formulations for Stored-Product and Food-Industry Pest Control. Insects 2021, 12, 590. [CrossRef] 
12. Johansson, S.G.O.; van Hage-Hamsten, M.; Johansson, E.; Wirén, A. The Storage Mite Story. In New Trends in Allergy III; Springer: Berlin/Heidelberg, Germany, 1991; pp. 439-446.

13. Collins, D. A review of alternatives to organophosphorus compounds for the control of storage mites. J. Stored Prod. Res. 2006, 42, 395-426. [CrossRef]

14. Li, C.; Zhan, X.; Sun, E.; Zhao, J.; Wang, H.; He, J.; Wang, J. The density and species of mite breeding in the stored products in China. Nutr. Hosp. 2015, 31, 798-807.

15. Tratwal, A.; Strażyński, P.; Mrówczyński, M. Metodyka integrowanej ochrony mieszanek zbożowych dla doradców. Inst. Ochr. Roślin Państwowy Inst. Badawczy. 2018, 1, 1-202.

16. Hubert, J.; Stejskal, V.; Munzbergova, Z.; Hajslova, J.; Arthur, F.H. Toxicity and efficacy of selected pesticides and new acaricides to stored product mites (Acari: Acaridida). Exp. Appl. Acarol. 2007, 42, 283-290. [CrossRef] [PubMed]

17. Dekeyser, M.A.; Downer, R.G. Biochemical and physiological targets for miticides. Pestic. Sci. 1994, 40, 85-101. [CrossRef]

18. Conyers, S.T.; Bell, C.H. The effect of modified atmospheres on the survival of the eggs of four storage mite species. Exp. Appl. Acarol. 2003, 31, 115-130. [CrossRef]

19. Ong, G.S.; Somerville, C.P.; Jones, T.W.; Walsh, J.P. Anaphylaxis triggered by benzyl benzoate in a preparation of depot testosterone undecanoate. Case Rep. Med. 2012, 2012, 384054. [CrossRef]

20. Stará, J.; Nesvorná, M.; Hubert, J. The toxicity of selected acaricides against five stored product mites under laboratory assay. J. Pest Sci. 2011, 84, 387-391. [CrossRef]

21. Kim, E.-H.; Kim, H.-K.; Choi, D.-H.; Ahn, Y.-J. Acaricidal activity of clove bud oil compounds against Tyrophagus putrescentiae (Acari: Acaridae). Appl. Entomol. Zool. 2003, 38, 261-266. [CrossRef]

22. Jeon, J.-H.; Park, J.-H.; Chung, N.; Lee, H.-S. Active monoterpene ketones isolated from Rosmarinus officinalis with fumigant and contact action against Tyrophagus putrescentiae (Schrank). J. Food Prot. 2014, 77, 1355-1360. [CrossRef]

23. Jiang, Z.; Akhtar, Y.; Bradbury, R.; Zhang, X.; Isman, M.B. Comparative toxicity of essential oils of Litsea pungens and Litsea cubeba and blends of their major constituents against the cabbage looper, Trichoplusia ni. J. Agric. Food Chem. 2009, 57, 4833-4837. [CrossRef]

24. Lee, H.-S. Chemical Composition and Acaricidal Effects of Essential Oils Extracted from Ligustrum japonicum against Acaridae and Pyroglyphid Mites. J. Appl. Biol. Chem. 2015, 58, 197-199. [CrossRef]

25. Ottoboni, F. House dust mites prevention in Italy. Boll. Zool Agr Bachic Ser. 2 1992, 24, 113-120.

26. Afifi, F.; Hafeez, S. Effect of different plant extracts on the toxicity and behaviour of Tyrophagus putrescentiae Schrank (Acari: Acaridae). Ann. Agric. Sci. Ain Shams Univ. 1988, 33, 1375-1385.

27. Song, H.Y.; Yang, J.Y.; Suh, J.W.; Lee, H.S. Acaricidal activities of apiol and its derivatives from Petroselinum sativum seeds against Dermatophagoides pteronyssinus, Dermatophagoides farinae, and Tyrophagus putrescentiae. J. Agric. Food Chem. 2011, 59, 7759-7764. [CrossRef]

28. Park, J.-H.; Lee, N.-H.; Yang, Y.-C.; Lee, H.-S. Food protective effects of 3-methylbenzaldehyde derived from Myosotis arvensis and its analogues against Tyrophagus putrescentiae. Sci. Rep. 2017, 7, 1-10. [CrossRef]

29. Alkot, W.M.; Naiem, E.S.; El-Ebiary, M.E.; Tayesh, M.A.A.; Seif, A.I. Lethal and sublethal effects of some essential oils against Callosobruchus maculatus, Tyrophagus putrescentiae and their natural enemies; Anisopteromalus calandrae and Cheyletus malaccensis. Egypt. J. Exp. Biol. 2020, 16, 33-45.

30. Satyal, P.; Craft, J.D.; Dosoky, N.S.; Setzer, W.N. The chemical compositions of the volatile oils of garlic (Allium sativum) and wild garlic (Allium vineale). Foods 2017, 6, 63. [CrossRef]

31. Mossa, A.-T.H.; Afia, S.I.; Mohafrash, S.M.; Abou-Awad, B.A. Formulation and characterization of garlic (Allium sativum L.) essential oil nanoemulsion and its acaricidal activity on eriophyid olive mites (Acari: Eriophyidae). Environ. Sci. Pollut. Res. 2018, 25, 10526-10537. [CrossRef] [PubMed]

32. Ndakidemi, B.; Mtei, K.; Ndakidemi, P.A. Impacts of synthetic and botanical pesticides on beneficial insects. Agric. Sci. 2016, 7, 364. [CrossRef]

33. De Assis, C.P.; Gondim, M.G., Jr.; De Siqueira, H.A.; Da Câmara, C.A. Toxicity of essential oils from plants towards Tyrophagus putrescentiae (Schrank) and Suidasia pontifica Oudemans (Acari: Astigmata). J. Stored Prod. Res. 2011, 47, 311-315. [CrossRef]

34. Raina, V.; Srivastava, S.; Aggarwal, K.; Ramesh, S.; Kumar, S. Essential oil composition of Cinnamomum zeylanicum Blume leaves from Little Andaman, India. Flavour Fragr. J. 2001, 16, 374-376. [CrossRef]

35. Perrucci, S. Acaricidal activity of some essential oils and their constituents against Tyrophagus longior, a mite of stored food. J. Food Prot. 1995, 58, 560-563. [CrossRef] [PubMed]

36. Hubert, J.; Münzbergová, Z.; Nesvorná, M.; Poltronieri, P.; Santino, A. Acaricidal effects of natural six-carbon and nine-carbon aldehydes on stored-product mites. Exp. Appl. Acarol. 2008, 44, 315-321. [CrossRef]

37. Sung, B.; Lim, J.; Lee, H. Food protective and color alteration effects of acaricidal aldehydes on Tyrophagus putrescentiae (Schrank). J. Food Prot. 2006, 69, 1728-1731. [CrossRef]

38. Ament, K.; Kant, M.R.; Sabelis, M.W.; Haring, M.A.; Schuurink, R.C. Jasmonic acid is a key regulator of spider mite-induced volatile terpenoid and methyl salicylate emission in tomato. Plant. Physiol. 2004, 135, 2025-2037. [CrossRef] [PubMed]

39. Smart, L.E.; Martin, J.L.; Limpalaër, M.; Bruce, T.J.; Pickett, J.A. Responses of herbivore and predatory mites to tomato plants exposed to jasmonic acid seed treatment. J. Chem. Ecol. 2013, 39, 1297-1300. [CrossRef] [PubMed] 
40. Macchioni, F.; Cioni, P.; Flamini, G.; Morelli, I.; Perrucci, S.; Franceschi, A.; Macchioni, G.; Ceccarini, L. Acaricidal activity of pine essential oils and their main components against Tyrophagus putrescentiae, a stored food mite. J. Agric. Food Chem. 2002, 50, 4586-4588. [CrossRef]

41. Lee, C.-H.; Park, J.-M.; Song, H.-Y.; Jeong, E.-Y.; Lee, H.-S. Acaricidal activities of major constituents of essential oil of Juniperus chinensis leaves against house dust and stored food mites. J. Food Prot. 2009, 72, 1686-1691. [CrossRef] [PubMed]

42. Khani, A.; Asghari, J. Insecticide activity of essential oils of Mentha longifolia, Pulicaria gnaphalodes and Achillea wilhelmsii against two stored product pests, the flour beetle, Tribolium castaneum, and the cowpea weevil, Callosobruchus maculatus. J. Insect Sci. 2012, 12, 1-10. [CrossRef]

43. Saleem, S.; Ali, Q.; Ali, K.; Majid, A. Toxicological and growth regulatory effects of acetone extract oils of indigenous medicinal plants against a stored grain pest, Cryptolestes ferrugineus (Stephens) (Coleoptera: Cucujidae). Pak. J. Zool 2016, 48, 903-906.

44. Tavares, W.; Freitas, S.; Grael, C.; Menezes, C.; Pereira, A.I.; Assis Júnior, S.; Grazziotti, G.; Zanuncio, J. Tenebrio molitor (Coleoptera: Tenebrionidae) as a guinea pig for the analysis of the toxicity of natural products. Vie Milieu 2013, 63, $193-204$.

45. Talukder, D.; Khanam, L.M. Toxicity of four plant based products against three stored product pests. J. Bio Sci. 2009, 17, 149-153. [CrossRef]

46. Lee, H.E.; Hong, S.J.; Hasan, N.; Baek, E.J.; Kim, J.T.; Kim, Y.D.; Park, M.K. Repellent efficacy of essential oils and plant extracts against Tribolium castaneum and Plodia interpunctella. Entomol. Res. 2020, 50, 450-459. [CrossRef]

47. Klys, M.; Przystupinska, A. The mortality of Oryzaephilus surinamensis Linnaeus, 1758 (Coleoptera: Silvanidae) induced by powdered plants. J. Plant. Prot. Res. 2015, 55, 110-116. [CrossRef]

48. Plata-Rueda, A.; Martínez, L.C.; Dos Santos, M.H.; Fernandes, F.L.; Wilcken, C.F.; Soares, M.A.; Serrão, J.E.; Zanuncio, J.C. Insecticidal activity of garlic essential oil and their constituents against the mealworm beetle, Tenebrio molitor Linnaeus (Coleoptera: Tenebrionidae). Sci. Rep. 2017, 7, 1-11. [CrossRef]

49. Yang, F.L.; Zhu, F.; Lei, C.L. Garlic essential oil and its major component as fumigants for controlling Tribolium castaneum (Herbst) in chambers filled with stored grain. J. Pest. Sci. 2010, 83, 311-317. [CrossRef]

50. Wang, Y.; You, C.X.; Yang, K.; Chen, R.; Zhang, W.J.; Wu, Y.; Liu, Z.L.; Du, S.S.; Deng, Z.W. Chemical constituents and insecticidal activities of the essential oil from Alpinia blepharocalyx rhizomes against Lasioderma serricorne. J. Serb. Chem. Soc. 2015, 80, 171-178, [CrossRef]

51. Guo, S.-S.; You, C.-X.; Liang, J.-Y.; Zhang, W.-J.; Yang, K.; Geng, Z.-F.; Wang, C.-F.; Du, S.-S.; Lei, N. Essential oil of Amomum maximum Roxb. and its bioactivities against two stored-product insects. J. Oleo Sci. 2015, 64, 1307-1314. [CrossRef]

52. Wang, Y.; You, C.X.; Wang, C.F.; Yang, K.; Chen, R.; Zhang, W.J.; Du, S.S.; Geng, Z.F.; Deng, Z.W. Chemical constituents and insecticidal activities of the essential oil from Amomum tsaoko against two stored-product insects. J. Oleo Sci. 2014, 63, 1019-1026. [CrossRef]

53. Rosa, J.S.; Oliveira, L.; Sousa, R.M.O.F.; Escobar, C.B.; Fernandes-Ferreira, M. Bioactivity of some Apiaceae essential oils and their constituents against Sitophilus zeamais (Coleoptera: Curculionidae). Bull. Entomol. Res. 2020, 110, 406-416. [CrossRef]

54. Chen, H.; Akinkurolere, R.; Zhang, H. Fumigant activity of plant essential oil from Armoracia rusticana (L.) on Plodia interpunctella (Lepidoptera: Pyralidae) and Sitophilus zeamais (Coleoptera: Curculionidae). Afr. J. Biotechnol. 2011, 10, 597-601.

55. Liang, J.-Y.; Wang, W.-T.; Zheng, Y.-F.; Zhang, D.; Wang, J.-L.; Guo, S.-S.; Zhang, W.-J.; Du, S.-S.; Zhang, J. Bioactivities and Chemical Constituents of Essential Oil Extracted from Artemisia anethoides Against Two Stored Product Insects. J. Oleo Sci. 2017, 66, 71-76. [CrossRef]

56. Bachrouch, O.; Ferjani, N.; Haouel, S.; Jemâa, J.M.B. Major compounds and insecticidal activities of two Tunisian Artemisia essential oils toward two major coleopteran pests. Ind. Crop. Prod. 2015, 65, 127-133. [CrossRef]

57. Abdelgaleil, S.A.M.; Mohamed, M.I.E.; Shawir, M.S.; Abou-Taleb, H.K. Chemical composition, insecticidal and biochemical effects of essential oils of different plant species from Northern Egypt on the rice weevil, Sitophilus oryzae L. J. Pest. Sci. 2016, 89, 219-229. [CrossRef]

58. Song, J.; Lee, H.-S. Insecticidal effects of Anethum graveolens and Artemisia vulgaris oils against Sitophilus zeamais and Sitotroga cerealella in the granary. J. Appl. Biol. Chem. 2017, 60, 1-3. [CrossRef]

59. Zhang, W.J.; Yang, K.; You, C.X.; Wang, Y.; Wang, C.F.; Wu, Y.; Geng, Z.F.; Su, Y.; Du, S.S.; Deng, Z.W. Bioactivity of essential oil from Artemisia stolonifera (Maxim.) komar. and its main compounds against two stored-product insects. J. Oleo Sci. 2015, 64, 299-307. [CrossRef]

60. Chu, S.S.; Liu, S.L.; Liu, Q.Z.; Jiang, G.H.; Liu, Z.L. Chemical composition and insecticidal activities of the essential oil of the flowering aerial parts of Aster ageratoides. J. Serb. Chem. Soc. 2013, 78, 209. [CrossRef]

61. Yang, K.; You, C.X.; Wang, C.F.; Lei, N.; Guo, S.S.; Geng, Z.F.; Du, S.S.; Ma, P.; Deng, Z.W. Chemical composition and bioactivity of essential oil of Atalantia guillauminii against three species stored product insects. J. Oleo Sci. 2015, 64, 1101-1109. [CrossRef]

62. Adarkwah, C.; Obeng-Ofori, D.; Büttner, C.; Reichmuth, C.; Schöller, M. Bio-rational control of red flour beetle Tribolium castaneum (Herbst)(Coleoptera: Tenebrionidae) in stored wheat with Calneem ${ }^{\circledR}$ oil derived from neem seeds. J. Pest Sci. 2010, 83, 471-479. [CrossRef]

63. Derbalah, A.S. Efficacy of some botanical extracts against Trogoderma granarium in wheat grains with toxicity evaluation. Sci. World J. 2012, 2012, 639854. [CrossRef] [PubMed]

64. Tawfeek, M.E.; Abu-Shall, A.M.H.; Gad, A.A.; Mohey, M. Evaluation of six plant essential oils against three stored product insects and their effects on the Haemogram under laboratory conditions. Alex. J. Agric. Sci. 2017, 62, 291-301. [CrossRef] 
65. Ziaee, M.; Moharramipour, S.; Mohsenifar, A. Toxicity of Carum copticum essential oil-loaded nanogel against Sitophilus granarius and Tribolium confusum. J. Appl. Entomol. 2014, 138, 763-771. [CrossRef]

66. Chu, S.S.; Liu, O.; Zhou, L.; Du, S.S.; Liu, Z.L. Chemical composition and toxic activity of essential oil of Caryopteris incana against Sitophilus zeamais. Afr. J. Biotechnol. 2011, 10, 8476-8480.

67. Keszthelyi, S.; Hoffmann, R.; Pónya, Z.; Pál-Fám, F. Acute and persistence effects of oil of Hippophae rhamnoides and Calendula officinalis on Sitophilus granarius (Coleoptera: Curculionidae) in stored maize. Acta Phytopathol. Entomol. Hung. 2017, 52, 255-264. [CrossRef]

68. Shah, M.; Prodhan, M.; Siddquie, M.; Mamun, M.; Shahjahan, M. Repellent effect of some indigenous plant extracts against saw-toothed grain beetle, Oryzaephilus surinamensis (L.). Int. J. Sustain. Crop Prod. 2008, 3, 51-54.

69. Liu, Z.L.; Yang, K.; Huang, F.; Liu, Q.Z.; Zhou, L.; Du, S.S. Chemical composition and toxicity of the essential oil of Cayratia japonica against two grain storage insects. J. Essent. Oil Res. 2012, 24, 237-240. [CrossRef]

70. Kachhwaha, N.; Meena, D.; Meena, S. Plant extracts controls Oryzaephilus surinamensis by showing repellency behavior. Eur. J. Exp. Biol. 2015, 5, 98-101.

71. Martínez, L.C.; Plata-Rueda, A.; Colares, H.C.; Campos, J.M.; Dos Santos, M.H.; Fernandes, F.L.; Serrão, J.E.; Zanuncio, J.C. Toxic effects of two essential oils and their constituents on the mealworm beetle, Tenebrio molitor. Bull. Entomol. Res. 2018, 108, 716-725. [CrossRef]

72. Luo, C.; Li, D.; Wang, Y.; Guo, S.; Zhang, D.; Du, S. Chemical Composition and Insecticide Efficacy of Essential Oils from Citrus medica L. var. sarcodactylis Swingle Against Tribolium castaneum Herbst in Stored Medicinal Materials. J. Essent. Oil Bear. Plants 2019, 22, 1182-1194. [CrossRef]

73. Abbas, S.; Farooq, A.; Muhammad, S.; Muhammad, Y.; Saeed, A.; Wali, M. Insecticidal and growth inhibition activities of Citrus paradisi and Citrus reticulata essential oils against lesser grain borer, Rhyzopertha dominica (F.) (Coleoptera: Bostrichidae). World J. Zool. 2012, 7, 289-294.

74. Zaka, S.M.; Iqbal, N.; Saeed, Q.; Akrem, A.; Batool, M.; Khan, A.A.; Anwar, A.; Bibi, M.; Azeem, S.; Bibi, R. Toxic effects of some insecticides, herbicides, and plant essential oils against Tribolium confusum Jacquelin du val (Insecta: Coleoptera: Tenebrionidae). Saudi J. Biol. Sci. 2019, 26, 1767-1771. [CrossRef]

75. Iram, N.; Arshad, M.; Akhter, N. Evaluation of botanical and synthetic insecticide for the control of Tribolium castaneum (Herbst)(Coleoptera: Tenebrionidae). Bio Assay 2013, 8, 1-10.

76. Saleem, M.; Hussain, D.; Rashid, R.H.; Saleem, H.M.; Ghouse, G.; Abbas, M. Insecticidal activities of two citrus oils against Tribolium castaneum (herbst). Am. J. Res. Commun. 2013, 1, 67-74.

77. Lü, J. Effect of Citrus reticulata Blanco essential oil on Cryptolestes ferrugineus (Stephens) adults. J. Food Prot. 2017, 80, 2090-2093. [CrossRef] [PubMed]

78. Campolo, O.; Romeo, F.V.; Malacrinò, A.; Laudani, F.; Carpinteri, G.; Fabroni, S.; Rapisarda, P.; Palmeri, V. Effects of inert dusts applied alone and in combination with sweet orange essential oil against Rhyzopertha dominica (Coleoptera: Bostrichidae) and wheat microbial population. Ind. Crop. Prod. 2014, 61, 361-369. [CrossRef]

79. Kim, S.-I.; Lee, D.-W. Toxicity of basil and orange essential oils and their components against two coleopteran stored products insect pests. J. Asia-Pac. Entomol. 2014, 17, 13-17. [CrossRef]

80. Popoviæ, A.; Šuæur, J.; Orèiæ, D.; Štrbac, P. Effects of essential oil formulations on the adult insect Tribolium castaneum (Herbst) (Coleoptera, Tenebrionidae). J. Cent. Eur. Agric. 2013, 14, 181-193. [CrossRef]

81. You, C.; Guo, S.; Zhang, W.; Yang, K.; Geng, Z.; Du, S.; Wang, C.; Deng, Z. Identification of repellent and insecticidal constituents from Artemisia mongolica essential oil against Lasioderma serricorne. J. Chem. 2015, 15, 1-7.

82. Rani, P.U. Fumigant and contact toxic potential of essential oils from plant extracts against stored product pests. J. Biopestic. 2012, 5, 120.

83. Polatoğlu, K.; Karakoç, Ö.C.; Yücel, Y.Y.; Gücel, S.; Demirci, B.; Başer, K.H.C.; Demirci, F. Insecticidal activity of edible Crithmum maritimum L. essential oil against Coleopteran and Lepidopteran insects. Ind. Crop. Prod. 2016, 89, 383-389. [CrossRef]

84. Bett, P.K.; Deng, A.L.; Ogendo, J.O.; Kariuki, S.T.; Kamatenesi-Mugisha, M.; Mihale, J.M.; Torto, B. Residual contact toxicity and repellence of Cupressus lusitanica Miller and Eucalyptus saligna Smith essential oils against major stored product insect pests. Ind. Crop. Prod. 2017, 110, 65-74. [CrossRef]

85. Franz, A.R.; Knaak, N.; Fiuza, L.M. Toxic effects of essential plant oils in adult Sitophilus oryzae (Linnaeus) (Coleoptera, Curculionidae). Rev. Bras. Entomol. 2011, 55, 116-120. [CrossRef]

86. Bossou, A.D.; Ahoussi, E.; Ruysbergh, E.; Adams, A.; Smagghe, G.; De Kimpe, N.; Avlessi, F.; Sohounhloue, D.C.; Mangelinckx, S. Characterization of volatile compounds from three Cymbopogon species and Eucalyptus citriodora from Benin and their insecticidal activities against Tribolium castaneum. Ind. Crop. Prod. 2015, 76, 306-317. [CrossRef]

87. Ukeh, D.A.; Oku, E.E.; Udo, I.A.; Nta, A.I.; Ukeh, J.A. Insecticidal effect of fruit extracts from Xylopia aethiopica and Dennettia tripetala (Annonaceae) against Sitophilus oryzae (Coleoptera: Curculionidae). Chil. J. Agric. Res. 2012, 72, 195. [CrossRef]

88. Chu, S.S.; Liu, S.L.; Liu, Q.Z.; Liu, Z.L.; Du, S.S. Composition and toxicity of Chinese Dracocephalum moldavica (Labiatae) essential oil against two grain storage insects. J. Med. Plants Res. 2011, 5, 5262-5267.

89. Zapata, N.; Smagghe, G. Repellency and toxicity of essential oils from the leaves and bark of Laurelia sempervirens and Drimys winteri against Tribolium castaneum. Ind. Crop. Prod. 2010, 32, 405-410. [CrossRef] 
90. Yang, K.; Guo, S.S.; Geng, Z.F.; Du, S.S.; Wang, C.F.; Deng, Z.W. Contact Toxicity and Repellency of the Essential Oil of Dictamnus dasycarpus Roots from China against Two Stored-Product Insects. Chem. Biodivers. 2015, 12, 980-986. [CrossRef] [PubMed]

91. Guo, S.-S.; You, C.-X.; Liang, J.-Y.; Zhang, W.-J.; Geng, Z.-F.; Wang, C.-F.; Du, S.-S.; Lei, N. Chemical composition and bioactivities of the essential oil from Etlingera yunnanensis against two stored product insects. Molecules 2015, 20, 15735-15747. [CrossRef] [PubMed]

92. El-Bakry, A.; Abdel-Aziz, N.; Sammour, E.; Abdelgaleil, S. Insecticidal activity of natural plant essential oils against some stored product insects and their side effects on wheat seed germination. Egypt. J. Biol. Pest. Control. 2016, $26,83$.

93. Negahban, M.; Moharramipour, S. Fumigant toxicity of Eucalyptus intertexta, Eucalyptus sargentii and Eucalyptus camaldulensis against stored-product beetles. J. Appl. Entomol. 2007, 131, 256-261. [CrossRef]

94. Parsia Aref, S.; Valizadegan, O.; Farashiani, M.E. The Insecticidal Effect of Essential Oil of Eucalyptus floribundi Against Two Major Stored Product Insect Pests; Rhyzopertha dominica (F.) and Oryzaephilus surinamensis (L.). J. Essent. Oil Bear. Plants 2016, 19 , 820-831. [CrossRef]

95. Ebadollahi, A.; Safaralizadeh, M.; Pourmirza, A. Fumigant toxicity of Lavandula stoechas L. oil against three insect pests attacking stored products. J. Plant. Prot. Res. 2010, 50, 56-60. [CrossRef]

96. Kambouzia, J.; Negahban, M.; Moharramipour, S. Fumigant toxicity of Eucalyptus leucoxylon against stored product insects. Am. -Eurasian J. Sustain. Agric. 2009, 3, 229-234.

97. Nouri-Ganbalani, G.; Ebadollahi, A.; Nouri, A. Chemical composition of the essential oil of Eucalyptus procera Dehnh. and Its insecticidal effects against two stored product insects. J. Essent. Oil Bear. Plants 2016, 19, 1234-1242. [CrossRef]

98. Tripathi, A.K.; Upadhyay, S. Repellent and insecticidal activities of Hyptis suaveolens (Lamiaceae) leaf essential oil against four stored-grain coleopteran pests. Int. J. Trop. Insect Sci. 2009, 29, 219-228. [CrossRef]

99. Guo, S.; Zhang, W.; Liang, J.; You, C.; Geng, Z.; Wang, C.; Du, S. Contact and repellent activities of the essential oil from Juniperus formosana against two stored product insects. Molecules 2016, 21, 504. [CrossRef]

100. Khani, A.; Rashid, B.; Mirshekar, A. Chemical composition and insecticidal efficacy of Juniperus polycarpus and Juniperus sabina essential oils against Tribolium confusum (Coleoptera: Tenebrionidae). Int. J. Food Prop. 2017, 20, 1221-1229. [CrossRef]

101. Li, H.Q.; Qi Bai, C.; Chu, S.S.; Zhou, L.; Du, S.S.; Liu, Z.L.; Liu, Q.Z. Chemical composition and toxicities of the essential oil derived from Kadsura heteroclita stems against Sitophilus zeamais and Meloidogyne incognita. J. Med. Plants Res. 2011, 5, $4943-4948$.

102. Torres, C.; Silva, G.; Tapia, M.; Rodríguez, J.C.; Figueroa, I.; Lagunes, A.; Santillán, C.; Robles, A.; Aguilar, S.; Ticuch, I. Insecticidal activity of Laurelia sempervirens (Ruiz \& Pav.) Tul. essential oil against Sitophilus zeamais Motschulsky. Chil. J. Agric. Res. 2014, 74, 421-426.

103. Jemâa, J.M.-B.; Tersim, N.; Khouja, M.L. Composition and repellent efficacy of essential oil from Laurus nobilis against adults of the cigarette beetle Lasioderma serricorne (Coleoptera: Anobiidae). Tunis. J. Plant Prot. 2011, 6, $29-41$.

104. Mediouni Ben Jemâa, J.; Tersim, N.; Toudert, K.T.; Khouja, M.L. Insecticidal activities of essential oils from leaves of Laurus nobilis L. from Tunisia, Algeria and Morocco, and comparative chemical composition. J. Stored Prod. Res. 2012, 48, 97-104. [CrossRef]

105. Guo, S.S.; Zhang, W.J.; You, C.X.; Liang, J.Y.; Yang, K.; Geng, Z.F.; Du, S.S.; Wang, C.F. Chemical composition of essential oil extracted from Laggera pterodonta and its bioactivities against two stored product insects. J. Food Process. Preserv. 2017, $41,12941$. [CrossRef]

106. Germinara, G.S.; Di Stefano, M.G.; De Acutis, L.; Pati, S.; Delfine, S.; De Cristofaro, A.; Rotundo, G. Bioactivities of Lavandula angustifolia essential oil against the stored grain pest Sitophilus granarius. Bull. Insectol. 2017, 70, 129-138.

107. Yang, K.; Wang, C.F.; You, C.X.; Geng, Z.F.; Sun, R.Q.; Guo, S.S.; Du, S.S.; Liu, Z.L.; Deng, Z.W. Bioactivity of essential oil of Litsea cubeba from China and its main compounds against two stored product insects. J. Asia Pac. Entomol. 2014, 17, 459-466. [CrossRef]

108. Ko, K.; Juntarajumnong, W.; Chandrapatya, A. Insecticidal Activities of Essential Oils from Fruits of Litsea salicifolia Roxb. ex Wall. Against Sitophilus zeamais Motschulsky and Tribolium castaneum (Herbst). Pak. J. Zool. 2010, 42, 551-557.

109. Kamanula, J.F.; Belmain, S.R.; Hall, D.R.; Farman, D.I.; Goyder, D.J.; Mvumi, B.M.; Masumbu, F.F.; Stevenson, P.C. Chemical variation and insecticidal activity of Lippia javanica (Burm. f.) Spreng essential oil against Sitophilus zeamais Motschulsky. Ind. Crop. Prod. 2017, 110, 75-82. [CrossRef]

110. Lima, R.K.; Cardoso, M.d.G.; Moraes, J.C.; Carvalho, S.M.; Rodrigues, V.G.; Guimarães, L.G.L. Chemical composition and fumigant effect of essentialoil of Lippia sidoides Cham. and monoterpenes against Tenebrio molitor (L.) (Coleoptera: Tenebrionidae). Ciênc. Agrotecnol. 2011, 35, 664-671. [CrossRef]

111. Wu, Y.; Zhang, W.-J.; Wang, P.-J.; Yang, K.; Huang, D.-Y.; Wei, J.-Y.; Tian, Z.-F.; Bai, J.-F.; Du, S.-S. Contact toxicity and repellency of the essential oil of Liriope muscari (DECN.) Bailey against three insect tobacco storage pests. Molecules 2015, 20, 1676-1685. [CrossRef]

112. Pang, X.; Feng, Y.X.; Qi, X.J.; Wang, Y.; Almaz, B.; Xi, C.; Du, S.S. Toxicity and repellent activity of essential oil from Mentha piperita Linn. leaves and its major monoterpenoids against three stored product insects. Environ. Sci. Pollut. Res. 2020, $27,7618-7627$. [CrossRef] [PubMed]

113. Khani, M.; Muhamad Awang, R.; Omar, D. Insecticidal Effects of Peppermint and Black Pepper Essential Oils against Rice Weevil, Sitophilus oryzae L. and Rice Moth, Corcyra cephalonica (St.). J. Med. Plants 2012, 11, 97-110.

114. Odeyemi, O.; Masika, P.; Afolayan, A. Insecticidal activities of essential oil from the leaves of Mentha longifolia L. subsp. capensis against Sitophilus zeamais (Motschulsky) (Coleoptera: Curculionidae). Afr. Entomol. 2008, 16, 220-225. [CrossRef] 
115. Mahmoudvand, M.; Abbasipour, H.; Basij, M.; Hosseinpour, M.H.; Rastegar, F.; Nasiri, M.B. Fumigant toxicity of some essential oils on adults of some stored-product pests. Chil. J. Agric. Res. 2011, 1, 71. [CrossRef]

116. Salem, N.; Bachrouch, O.; Sriti, J.; Msaada, K.; Khammassi, S.; Hammami, M.; Selmi, S.; Boushih, E.; Koorani, S.; Abderraba, M.; et al. Fumigant and repellent potentials of Ricinus communis and Mentha pulegium essential oils against Tribolium castaneum and Lasioderma serricorne. Int. J. Food Prop. 2017, 20, 2899-2913. [CrossRef]

117. Yıldırım, E.; Kesdek, M.; Aslan, İ.; Çalmaşur, Ö.; Şahin, F. The effects of essential oils from eight plant species on two pests of stored product insects. Fresen. Environ. Bull. 2005, 14, $23-27$.

118. Herrera, J.M.; Zunino, M.P.; Massuh, Y.; Pizzollito, R.; Dambolena, J.S.; Gañan, N.A.; Zygadlo, J.A. Fumigant toxicity from five essential oils rich in ketones against Sitophilus zeamais (Motschulsky). Agriscientia 2014, 31, 35-41. [CrossRef]

119. Chen, X.B.; Chen, R.; Luo, Z.R. Chemical composition and insecticidal properties of essential oil from aerial parts of Mosla soochowensis against two grain storage insects. Trop. J. Pharm. Res. 2017, 16, 905-910. [CrossRef]

120. Feng, Y.X.; Wang, Y.; Geng, Z.F.; Zhang, D.; Almaz, B.; Du, S.S. Contact toxicity and repellent efficacy of Valerianaceae spp. to three stored-product insects and synergistic interactions between two major compounds camphene and bornyl acetate. Ecotoxicol. Environ. Saf. 2020, 190, 110106. [CrossRef] [PubMed]

121. Hossain, F.; Lacroix, M.; Salmieri, S.; Vu, K.; Follett, P.A. Basil oil fumigation increases radiation sensitivity in adult Sitophilus oryzae (Coleoptera: Curculionidae). J. Stored Prod. Res. 2014, 59, 108-112. [CrossRef]

122. Saleem, M.B.; Hussain, M.; Hussain, M.; Awais, M.; Hayat, M.M.U.; Zulifqar, M.W.; Khadim, M.M. Efficacy of different plant extract on packaging materials against Tribolium castaneum and Trogoderma granarium. RND J. 2018, 54, 469-478.

123. Ogendo, J.O.; Kostyukovsky, M.; Ravid, U.; Matasyoh, J.C.; Deng, A.L.; Omolo, E.O.; Kariuki, S.T.; Shaaya, E. Bioactivity of Ocimum gratissimum L. oil and two of its constituents against five insect pests attacking stored food products. J. Stored Prod. Res. 2008, 44, 328-334. [CrossRef]

124. Çağlar, Ö.; Çalmaşur, Ö.; Aslan, I.; Kaya, O. Insecticidal effect of essential oil of Origanum acutidens against several stored product pests. Fresenius Environ. Bull. 2007, 16, 1395-1400.

125. Demirel, N.; Kurt, S.; Gunes, U.; Uluc, F.T.; Cabuk, F. Toxicological responses of confused flour beetle, Tribolium confusum du Val (Coleoptera: Tenebrionoidea) to various isothiocyanate compounds. Asian J. Chem 2009, 21, 6411-6416.

126. Zhang, H.M.; Guo, S.S.; Fan, B.; Du, S.S.; Wang, Y.Y.; Deng, Z.W. Evaluation of efficacy of the essential oil from Ostericum viridiflorum (Turcz.) Kitagawa in control of stored product insects. Environ. Sci. Pollut. Res. 2019, 26, 1406-1413. [CrossRef]

127. You, C.x.; Wang, Y.; Zhang, W.J.; Yang, K.; Wu, Y.; Geng, Z.F.; Chen, H.P.; Jiang, H.Y.; Du, S.S.; Deng, Z.W.; et al. Chemical constituents and biological activities of the Purple perilla essential oil against Lasioderma serricorne. Ind. Crop. Prod. 2014, 61, 331-337. [CrossRef]

128. Hashemi, S.M.; Safavi, S.A. Chemical constituents and toxicity of essential oils of oriental arborvitae, Platycladus orientalis (L.) Franco, against three stored-product beetles. Chil. J. Agric. Res. 2012, 72, 188. [CrossRef]

129. Maede, M.; Hamzeh, I.; Hossein, D.; Majid, A.; Reza, R.K. Bioactivity of essential oil from Satureja hortensis (Laminaceae) against three stored-product insect species. Afr. J. Biotechnol. 2011, 10, 6620-6627.

130. Spochacz, M.; Chowański, S.; Szymczak, M.; Lelario, F.; Bufo, S.A.; Adamski, Z. Sublethal effects of Solanum nigrum fruit extract and its pure glycoalkaloids on the physiology of Tenebrio molitor (Mealworm). Toxins 2018, 10, 504. [CrossRef]

131. Krishna, A.; Prajapati, V.; Bhasney, S.; Tripathi, A.K.; Kumar, S. Potential toxicity of new genotypes of Tagetes (Asteraceae) species against stored grain insect pests. Int. J. Trop. Insect Sci. 2005, 25, 122-128. [CrossRef]

132. Khani, A.; Heydarian, M. Fumigant and repellent properties of sesquiterpene-rich essential oil from Teucrium polium subsp. capitatum (L.). Asian Pac. J. Trop. Med. 2014, 7, 956-961. [CrossRef]

133. Ngassoum, M.B.; Tinkeu, L.S.N.; Ngatanko, I.; Tapondjou, L.A.; Lognay, G.; Malaisse, F.; Hance, T. Chemical composition, insecticidal effect and repellent activity of essential oils of three aromatic plants, alone and in combination, towards Sitophilus oryzae L. (Coleoptera: Curculionidae). Nat. Prod. Commun. 2007, 2, 1229-1232. [CrossRef]

134. Khoshnoud, H.; Ghiyasi, M.; Amirnia, R.; Fard, S.S.; Tajbakhsh, M.; Salehzadeh, H.; Alahyary, P. The potential of using insecticidal properties of medicinal plants against insect pests. Pak. J. Biol. Sci. PJBS 2008, 11, 1380-1384. [CrossRef] [PubMed]

135. Zhang, W.-J.; Zhang, Z.; Chen, Z.-Y.; Liang, J.-Y.; Geng, Z.-F.; Guo, S.-S.; Du, S.-S.; Deng, Z.-W. Chemical composition of essential oils from six Zanthoxylum species and their repellent activities against two stored-product insects. J. Chem. 2017, 71, 83-89.

136. Wang, Y.; Zhang, L.T.; Feng, Y.X.; Guo, S.S.; Pang, X.; Zhang, D.; Geng, Z.F.; Du, S.S. Insecticidal and repellent efficacy against stored-product insects of oxygenated monoterpenes and 2-dodecanone of the essential oil from Zanthoxylum planispinum var. dintanensis. Environ. Sci. Pollut. Res. 2019, 26, 24988-24997. [CrossRef] [PubMed]

137. Wang, Y.; You, C.; Yang, K.; Wu, Y.; Chen, R.; Zhang, W.; Liu, Z.; Du, S.; Deng, Z.; Geng, Z. Bioactivity of essential oil of Zingiber purpureum rhizomes and its main compounds against two stored product insects. J. Econ. Entomol. 2015, 108, 925-932. [CrossRef] [PubMed]

138. Dwivedi, S.; Garg, S. Efficacy of plant extracts as repellent against rice moth, Corcyra cephalonica(stainton). Pestology 1999, 23, 31-33.

139. Iqbal, J.; Jilani, G.; Aslam, M. Growth inhibiting effects of plant extracts against the grain moth, Sitotroga cerealella (Oliv.) (Gelechiidae: Lepidoptera). Pak. J. Zool. 2010, 42, 597-601.

140. Adeyemo, A.C.; Ashamo, M.O.; Odeyemi, O.O. Aframomum melegueta: A potential botanical pesticide against Sitotroga cerealella infestation on two paddy varieties. Arch. Phytopathol. Plant. Prot. 2014, 47, 1841-1851. [CrossRef] 
141. Bouayad, N.; Rharrabe, K.; Ghailani, N.; Jbilou, R.; Domínguez, P.C. Insecticidal effects of Moroccan plant extracts on development, energy reserves and enzymatic activities of Plodia interpunctella. Span. J. Agric. Res. 2013, 189-198. [CrossRef]

142. Lee, S.H.; Chang, Y.; Na, J.H.; Han, J. Development of anti-insect multilayered films for brown rice packaging that prevent Plodia interpunctella infestation. J. Stored Prod. Res. 2017, 72, 153-160. [CrossRef]

143. Yang, F.L.; Zhu, F.; Lei, C.L. Insecticidal activities of garlic substances against adults of grain moth, Sitotroga cerealella (Lepidoptera: Gelechiidae). Insect Sci. 2012, 19, 205-212. [CrossRef]

144. Shah, S.; Ma, M.; Ali, A.; Kaya, M.; Li, X.-G.; Wu, G.; Yang, F.-L. Effects of diallyl trisulfide, an active substance from garlic essential oil, on structural chemistry of chitin in Sitotroga cerealella (Lepidoptera: Gelechiidae). Pestic. Biochem. Physiol. 2021, 50, 79-85.

145. Wu, M.-Y.; Ying, Y.-Y.; Zhang, S.-S.; Li, X.-G.; Yan, W.-H.; Yao, Y.-C.; Shah, S.; Wu, G.; Yang, F.-L. Effects of diallyl trisulfide, an active substance from garlic essential oil, on energy metabolism in male moth Sitotroga cerealella (Olivier). Insects 2020, 11, 270. [CrossRef] [PubMed]

146. Chang, M.-M.; Shah, S.; Wu, M.-Y.; Zhang, S.-S.; Wu, G.; Yang, F.-L. Effect of diallyl trisulfide on the reproductive behavior of the grain moth, Sitotroga cerealella (Lepidoptera: Gelechiidae). Insects 2020, 11, 21. [CrossRef]

147. Işıkber, A.A.; Özder, N.; Sağlam, Ö. Susceptibility of eggs of Tribolium confusum, Ephestia kuehniella and Plodia interpunctella to four essential oil vapors. Phytoparasitica 2009, 37, 231. [CrossRef]

148. Sabbour, M.M.; Abd El-Aziz, S.E.-S. Impact of certain nano oils against Ephestia kuehniella and Ephestia cautella (LepidopteraPyralidae) under laboratory and store conditions. Bull. Natl. Res. Cent. 2019, 43, 1-7. [CrossRef]

149. Sabbour, M.M.; Abd El-Aziz, S.E.-S. The combined effect of Metarhizium anisopliae and some natural oils against Ephestia kuehniella and Ephestia cautella (Lepidoptera-Pyralidae) under laboratory and store conditions. Biosci. Res. 2018, 15, 3467-3476.

150. Akinneye, J.O.; Ogungbite, O.C. Effect of seed extracts of five indigenous plants against the stored product Moth, Ephestia cautella (Walker) (Lepidoptera: Pyralidae). Arch. Phytopathol. Plant. Prot. 2013, 46, 1488-1496. [CrossRef]

151. Akinneye, J.O.; Ogungbite, O.C. Entomotoxicant potential of some medicinal plant against Ephestia cautella infesting cocoa bean in storage. Int. J. Appl. Sci. Eng. 2016, 14, 59-68.

152. Karahroodi, Z.R.; Moharramipour, S.; Rahbarpour, A. Investigated repellency effect of some essential oils of 17 native medicinal plants on adults Plodia interpunctella. Am. Eurasian J. Sustain. Agric. 2009, 3, 181-185.

153. Mikhaiel, A. Potential of some volatile oils in protecting packages of irradiated wheat flour against Ephestia kuehniella and Tribolium castaneum. J. Stored Prod. Res. 2011, 47, 357-364. [CrossRef]

154. Parthiban, P.; Chinniah, C.; Kalyanasundarm, M.; Baskaran, R. Kairomonal effect of acetone extracts of groundnut on foraging activities of Trichogramma chilonis (Ishii) and Chrysoperla zastrowi sillemi (Esben-Peterson). Legume Res. An. Int. J. 2017, 40, 393-396. [CrossRef]

155. Shakarami, J.; Eftekharifar, R.; Latifian, M.; Jafari, S. Insecticidal activity and synergistic effect of Beauvaria bassiana (Bals.) Vuill. and three botanical compounds against third instar larvae of Ephestia kuehniella Zeller. Res. Crop. 2015, 16, 296-303. [CrossRef]

156. Bouzeraa, H.; Bessila-Bouzeraa, M.; Labed, N. Repellent and fumigant toxic potential of three essential oils against Ephestia kuehniella. Biosyst. Divers. 2019, 27, 349-353. [CrossRef]

157. Naseri, B.; Abedi, Z.; Abdolmaleki, A.; Jafary-Jahed, M.; Borzoui, E.; Mozaffar Mansouri, S. Fumigant toxicity and sublethal effects of Artemisia khorassanica and Artemisia sieberi on Sitotroga cerealella (Lepidoptera: Gelechiidae). J. Insect Sci. 2017, 17, 100. [CrossRef]

158. Borzoui, E.; Naseri, B.; Abedi, Z.; Karimi-Pormehr, M.S. Lethal and sublethal effects of essential oils from Artemisia khorassanica and Vitex pseudo-negundo against Plodia interpunctella (Lepidoptera: Pyralidae). Environ. Entomol. 2016, 45, 21. [CrossRef] [PubMed]

159. Sim, M.-J.; Choi, D.-R.; Ahn, Y.-J. Vapor phase toxicity of plant essential oils to Cadra cautella (Lepidoptera: Pyralidae). J. Econ. Entomol. 2006, 99, 593-598. [CrossRef] [PubMed]

160. Dubai, E.S.; Hamad, B.S.; Hanawi, M.J.; Swail, M.A. The Compatibility between the neem oil of Azadirachta indica and Bacillus thuringiensis in controlling fig moth (Ephestia Cautella). Indian J. Public Health Res. Dev. 2019, 10, 2455-2460. [CrossRef]

161. Ghasemi, V.; Yazdi, A.K.; Tavallaie, F.Z.; Sendi, J.J. Effect of essential oils from Callistemon viminalis and Ferula gummosa on toxicity and on the hemocyte profile of Ephestia kuehniella (Lep.: Pyralidae). Arch. Phytopathol. Plant. Prot. 2014, 47, 268-278. [CrossRef]

162. Bader, A.; Omran, Z.; Al-Asmari, A.I.; Santoro, V.; De Tommasi, N.; D’Ambola, M.; Dal Piaz, F.; Conti, B.; Bedini, S.; Halwani, M. Systematic phytochemical screening of different organs of Calotropis procera and the ovicidal effect of their extracts to the foodstuff pest Cadra cautella. Molecules 2021, 26, 905. [CrossRef]

163. Shahriari, M.; Sahbzadeh, N.; Zibaee, A.; Khani, A.; Senthil-Nathan, S. Metabolic response of Ephestia kuehniella Zeller (Lepidoptera: Pyralidae) to essential oil of Ajwain and thymol. Toxin Rev. 2017, 36, 204-209. [CrossRef]

164. Jalali, M.; Hosseininaveh, V.; Imani, S. Inhibitory activity of proteinaceous inhibitors from Cassia angustifolia and Trigonella foenum-graecum seeds against Plodia interpunctella (Lepidoptera: Pyralidae): Interaction of the inhibitors and the insect digestive enzymes. Arch. Phytopathol. Plant. Prot. 2015, 48, 268-276. [CrossRef]

165. Allahvaisi, S.; Maroufpoor, M.; Abdolmaleki, A.; Hoseini, S.-A.; Ghasemzadeh, S. The effect of plant oils for reducing contamination of stored packaged-foodstuffs. J. Plant. Prot. Res. 2011, 51, 82-86. [CrossRef]

166. Kim, J.; Yoon, C.S.; Lee, S.e.; Na, J.H.; Han, J. Development of insect-proof starch adhesive containing encapsulated cinnamon oil for paper box adhesion to inhibit Plodia interpunctella larvae infestation. J. Food Sci. 2020, 85, 3363-3371. [CrossRef] 
167. Song, A.Y.; Choi, H.Y.; Lee, E.S.; Han, J.; Min, S.C. Development of Anti-Insect Microencapsulated Polypropylene Films Using a Large Scale Film Coating System. J. Food Sci. 2018, 83, 1011-1016. [CrossRef]

168. Kim, I.H.; Song, A.Y.; Han, J.; Park, K.H.; Min, S.C. Indian meal moth (Plodia interpunctella)-resistant food packaging film development using microencapsulated cinnamon oil. J. Food Sci. 2014, 79, 2023-2030. [CrossRef]

169. Jo, H.J.; Park, K.M.; Min, S.C.; Na, J.H.; Park, K.H.; Han, J. Development of an anti-insect sachet using a polyvinyl alcoholcinnamon oil polymer strip against Plodia interpunctella. J. Food Sci. 2013, 78, 1713-1720. [CrossRef]

170. Song, J.-E.; Kim, J.-M.; Lee, N.-H.; Yang, J.-Y.; Lee, H.-S. Acaricidal and insecticidal activities of essential oils against a stored-food mite and stored-grain insects. J. Food Prot. 2016, 79, 174-178. [CrossRef] [PubMed]

171. Jesser, E.N.; Werdin-González, J.O.; Murray, A.P.; Ferrero, A.A. Efficacy of essential oils to control the Indian meal moth, Plodia interpunctella (Hübner) (Lepidoptera: Pyralidae). J. Asia Pac. Entomol. 2017, 20, 1122-1129. [CrossRef]

172. Ayvaz, A.; Karaborklu, S.; Sagdıc, O. Fumigant toxicity of five essential oils against the eggs of Ephestia kuehniella Zeller and Plodia interpunctella (Hübner) (Lepidoptera: Pyralidae). Asian J. Chem. 2009, 21, 596-604.

173. Karabörklü, S.; Ayvaz, A.; Yilmaz, S.; Akbulut, M. Chemical composition and fumigant toxicity of some essential oils against Ephestia kuehniella. J. Econ. Entomol. 2011, 104, 1212-1219. [CrossRef]

174. Adjalian, E.; Sessou, P.; Alitonou, G.A.; Noudogbessi, J.P.; Kossou, D.; Menut, C.; Sohounhloue, D. Ovicide and Larvicide Activities of the Essential Oils Extracted from Polyalthia longifolia and Clausena anisata on Sitotroga cerealella. Online J. Biol. Sci. 2017, 17, 193-200. [CrossRef]

175. Akinneye, J.; Adedire, C.; Arannilewa, S. Potential of Cleisthopholis patens Elliot as a maize protectant against the stored product moth, Plodia interpunctella (Hubner) (Lepidoptera; Pyralidae). Afr. J. Biotechnol. 2006, 5, 2510-2515.

176. Abbood, N.M.; Ali, S.T. The role of silver nanoparticles and some extracts of damas Conocarpus lancifolius in controlling stored dates moth Ephestia cautella (Walker). Plant. Arch. 2020, 20, 3301-3305.

177. Lee, M.-J.; Lee, S.-E.; Kang, M.-S.; Park, B.; Lee, S.-G.; Lee, H.-S. Acaricidal and insecticidal properties of Coriandrum sativum oils and their major constituents extracted by three different methods against stored product pests. Appl. Biol. Chem. 2018, 61, 481-488. [CrossRef]

178. Pascual-Villalobos, M.; López, M.; Castañé, C.; Soler, A.; Riudavets, J. Encapsulated essential oils as an alternative to insecticides in funnel traps. J. Econ. Entomol. 2015, 108, 411-419. [CrossRef]

179. Maroufpoor, M.; Ebadollahi, A.; Vafaee, Y.; Badiee, E. Chemical composition and toxicity of the essential oil of Coriandrum sativum L. and Petroselinum crispum L. against three Stored-Product insect pests. J. Essent. Oil Bear. Plants 2016, 19, 1993-2002. [CrossRef]

180. Tunc, I.; Berger, B.; Erler, F.E.; Dağl1, F. Ovicidal activity of essential oils from five plants against two stored-product insects. $J$. Stored Prod. Res. 2000, 36, 161-168. [CrossRef]

181. Bett, P.K.; Deng, A.L.; Ogendo, J.O.; Kariuki, S.T.; Kamatenesi-Mugisha, M.; Mihale, J.M.; Torto, B. Chemical composition of Cupressus lusitanica and Eucalyptus saligna leaf essential oils and bioactivity against major insect pests of stored food grains. Ind. Crop. Prod. 2016, 82, 51-62. [CrossRef]

182. Ulukanli, Z.; Karabörklü, S.; Ates, B.; Erdogan, S.; Cenet, M.; Karaaslan, M.G. Chemical composition of the essential oil from Cupressus sempervirens L. horizontalis resin in conjunction with its biological assessment. J. Essent. Oil Bear. Plants 2014, 17, 277-287. [CrossRef]

183. Bardón, A.; Popich, S.; Valdés, D.A.; Catalán, C.A. Toxic effects of a lactone-containing fraction of Cyrtocymura cincta (Asteraceae) on Sitotroga cerealella (Lepidoptera: Gelechiidae). J. Econ. Entomol. 1999, 92, 1369-1372. [CrossRef]

184. Fouad, H.A.; Faroni, L.R.D.A.; de Souza Tavares, W.; Ribeiro, R.C.; de Sousa Freitas, S.; Zanuncio, J.C. Botanical extracts of plants from the Brazilian Cerrado for the integrated management of Sitotroga cerealella (Lepidoptera: Gelechiidae) in stored grain. J. Stored Prod. Res. 2014, 57, 6-11. [CrossRef]

185. Shukla, S.; Tiwari, S. Insecticidal activity of Dryopteris filix-mas (Linn.) schott ethanolic extract against Corcyra cephalonica Staint. (Lepidoptera: Pyralidae). J. Biopestic. 2011, 11, 138.

186. Khan, S.U.; Khan, A.-U.; Shah, A.-U.-H.A.; Shah, S.M.; Hussain, S.; Ayaz, M.; Ayaz, S. Heavy metals content, phytochemical composition, antimicrobial and insecticidal evaluation of Elaeagnus angustifolia. Toxicol. Ind. Health 2016, 32, 154-161. [CrossRef]

187. Abbasipour, H.; Mahmoudvand, M.; Rastegar, F.; Hosseinpour, M.H. Fumigant toxicity and oviposition deterrency of the essential oil from cardamom, Elettaria cardamomum, against three stored-product insects. J. Insect Sci. 2011, 11, 165. [CrossRef] [PubMed]

188. Mahmoudvand, M.; Abbasipour, H.; Rastegar, F.; Hosseinpour, M.H.; Basij, M. Efficacy of some plants as a post-harvest protectant against some major stored pests. Arch. Phytopathol. Plant. Prot. 2012, 45, 806-811. [CrossRef]

189. Jemâa, J.M.B.; Haouel, S.; Bouaziz, M.; Khouja, M.L. Seasonal variations in chemical composition and fumigant activity of five Eucalyptus essential oils against three moth pests of stored dates in Tunisia. J. Stored Prod. Res. 2012, 48, 61-67. [CrossRef]

190. Park, J.-H.; Lee, H.-S. Toxicities of Eucalyptus dives oil, 3-carvomenthenone, and its analogues against stored-product insects. J. Food Prot. 2018, 81, 653-658. [CrossRef]

191. Tah, G.T.T.; Seri-Kouassi, B.; Aboua, L.R.N.; Koua, K.H. Insecticidal activity of essential oil of Eucalyptus platyphylla and Mentha piperita on cocoa bean stocks insects. Eur J. Sci Res. 2011, 49, 177-186.

192. Jesser, E.; Lorenzetti, A.S.; Yeguerman, C.; Murray, A.P.; Domini, C.; Werdin-González, J.O. Ultrasound assisted formation of essential oil nanoemulsions: Emerging alternative for Culex pipiens pipiens Say (Diptera: Culicidae) and Plodia interpunctella Hübner (Lepidoptera: Pyralidae) management. Ultrason. Sonochem. 2020, 61, 104832. [CrossRef] 
193. Rajopadhye, A.A.; Upadhye, A.S.; Taware, S. Bioactivity of indigenous plant Glossocardia bosvallia (Lf) DC. against insect pests of stored products. Indian J. Tradit. Knowl. 2016, 15, 260-265.

194. Gotyal, B.; Srivastava, C.; Walia, S. Fumigant Toxicity of Essential Oil from Lantana camara Against Almond Moth, Cadra cautella (Walker). J. Essent. Oil Bear. Plants 2016, 19, 1521-1526. [CrossRef]

195. Salehi, T.; Karimi, J.; Hasanshahi, G.; Askarianzadeh, A.; Abbasipour, H.; Garjan, A.S. The effect of essential oils from Laurus nobilis and Myrtus commonis on the adults of mediterranean flour moth, Ephestia kuehniella Zeller (Lep.: Pyralidae). J. Essent. Oil Bear. Plants 2014, 17, 553-561. [CrossRef]

196. Fadia, A.; Al-Naser, Z.; Al-Hakim, W. Chemical composition of Lavandula angustifolia Miller and Rosmarinus officinalis L. essential oils and fumigant toxicity against larvae of Ephestia kuehniella Zeller. Int. J. Chem. Technol. Res. 2015, 8, 1382-1390.

197. Zallaghi, N.; Ahmadi, M. Combined action of Lavandula angustifolia Miller essential oil and gamma irradiation treatment on some biological aspects of the Mediterranean flour moth Ephestia kuehniella (Zeller). Int. J. Pest. Manag. 2020, 67, 203-215. [CrossRef]

198. Corzo, F.L.; Traverso, L.; Sterkel, M.; Benavente, A.; Ajmat, M.T.; Ons, S. Plodia interpunctella (Lepidoptera: Pyralidae): Intoxication with essential oils isolated from Lippia turbinata (Griseb.) and analysis of neuropeptides and neuropeptide receptors, putative targets for pest control. Arch. Insect Biochem. Physiol. 2020, 104, 21684. [CrossRef] [PubMed]

199. Louni, M.; Shakarami, J.; Negahban, M. Insecticidal efficacy of nanoemulsion containing Mentha longifolia essential oil against Ephestia kuehniella (Lepidoptera: Pyralidae). J. Crop. Prot. 2018, 7, 171-182.

200. Allahvaisi, S.; Talebi, K.; Talebi, J.; Sohrab, I.; Khanjani, M. Contact toxicity of ploy lactic acid nanofibers loaded with two essential oils against Plodia interpunctella Hub. (Lepidoptera: Pyralidae). J. Biopestic. 2017, 10, 50-59.

201. Chaaban, S.B.; Hamdi, S.H.; Mahjoubi, K.; Jemâa, J.M.B. Composition and insecticidal activity of essential oil from Ruta graveolens, Mentha pulegium and Ocimum basilicum against Ectomyelois ceratoniae Zeller and Ephestia kuehniella Zeller (Lepidoptera: Pyralidae). J. Plant. Dis. Prot. 2019, 126, 237-246. [CrossRef]

202. Eliopoulos, P.; Hassiotis, C.; Andreadis, S.; Porichi, A.-E. Fumigant toxicity of essential oils from basil and spearmint against two major pyralid pests of stored products. J. Econ. Entomol. 2015, 108, 805-810. [CrossRef]

203. Aslan, İ.; Çalmaşur, Ö.; Şahin, F.; Çaglar, Ö. Insecticidal effects of essential plant oils against Ephestia kuehniella (Zell.), Lasioderma serricorne (F.) and Sitophilus granarius (L.). J. Plant. Dis. Prot. 2005, 112, 257-267.

204. Soni, R.; Sharma, G.; Jasuja, N.D. Essential oil yield pattern and antibacterial and insecticidal activities of Trachyspermum ammi and Myristica fragrans. Scientifica 2016, 3, 1428194.

205. Ayvaz, A.; Sagdic, O.; Karaborklu, S.; Ozturk, I. Insecticidal activity of the essential oils from different plants against three stored-product insects. J. Insect Sci. 2010, 10, 21. [CrossRef]

206. Jembere, B.; Obeng-Ofori, D.; Hassanali, A.; Nyamasyo, G. Products derived from the leaves of Ocimum kilimandscharicum (Labiatae) as post-harvest grain protectants against the infestation of three major stored product insect pests. Bull. Entomol. Res. 1995, 1, 1-26. [CrossRef]

207. Bekele, A.; Obeng-Ofori, D.; Hassanali, A. Evaluation of Ocimum suave (Willd) as a source of repellents, toxicants and protectants in storage against three stored product insect pests. Int. J. Pest Manag. 1996, 90, 52-57.

208. Prabu, S.; Jing, D.; Chandran, V.; Mathew, P. Insecticidal activity of Origanum majorana L. essential oil as anti-cholinergic agent. Entomol. Res. 2020, 50, 402-413. [CrossRef]

209. Taibi, F.; Boumendjel, M.; Moncef, Z.; Omar, S.; Taha, K.; Amel, D.; Safa, A.; Hassiba, R.; Hanène, C.; Nacira, S. Conservation of stored food using plant's extracts. Effect of oregano (Origanum vulgaris) essential oils on the reproduction and development of flour moth (Ephestia kuehniella). Cell. Mol. Biol. 2018, 64, 5-11. [CrossRef] [PubMed]

210. Ulukanli, Z.; Karabörklü, S.; Bozok, F.; Burhan, A.; Erdogan, S.; Cenet, M.; Karaaslan, M.G. Chemical composition, antimicrobial, insecticidal, phytotoxic and antioxidant activities of Mediterranean Pinus brutia and Pinus pinea resin essential oils. Chin. J. Nat. Med. 2014, 12, 901-910. [CrossRef]

211. Oh, H.-W.; Yun, C.-S.; Jeon, J.H.; Kim, J.-A.; Park, D.-S.; Ryu, H.W.; Oh, S.-R.; Song, H.-H.; Shin, Y.; Jung, C.S. Conifer diterpene resin acids disrupt juvenile hormone-mediated endocrine regulation in the indian meal moth, Plodia interpunctella. J. Chem. Ecol. 2017, 43, 703-711. [CrossRef]

212. Hematpoor, A.; Liew, S.Y.; Azirun, M.S.; Awang, K. Insecticidal activity and the mechanism of action of three phenylpropanoids isolated from the roots of Piper sarmentosum Roxb. Sci. Rep. 2017, 7, 1-13. [CrossRef]

213. Bachrouch, O.; Jemâa, J.M.-B.; Wissem, A.W.; Talou, T.; Marzouk, B.; Abderraba, M. Composition and insecticidal activity of essential oil from Pistacia lentiscus L. against Ectomyelois ceratoniae Zeller and Ephestia kuehniella Zeller (Lepidoptera: Pyralidae). J. Stored Prod. Res. 2010, 46, 242-247. [CrossRef]

214. Sabbour, M.; Abd El-Aziz, S.E. Roll of three essential oils and their Nano against Ephestia cautella (Lepidoptera-Pyralidae) under laboratory and store conditions. Int. J. PharmTech Res. 2016, 9, 194-200.

215. Sabbour, M.M.; Abd El-Aziz, S.E.-S. Screening effects of three natural oils and their nano against Ephestia kuehniella (LepidopteraPyralidae) in laboratory and store. Biosci. Res. 2017, 14, 408-416.

216. Mora, C.A.; Halter, J.G.; Adler, C.; Hund, A.; Anders, H.; Yu, K.; Stark, W.J. Application of the Prunus spp. cyanide seed defense system onto wheat: Reduced insect feeding and field growth tests. J. Agric. Food Chem. 2016, 64, 3501-3507. [CrossRef] [PubMed]

217. Moazeni, N.; Khajeali, J.; Izadi, H.; Mahdian, K. Chemical composition and bioactivity of Thymus daenensis Celak (Lamiaceae) essential oil against two lepidopteran stored-product insects. J. Essent. Oil Res. 2014, 26, 118-124. [CrossRef] 
218. Maedeh, M.; Hamzeh, I.; Hossein, D.; Majid, A.; Reza, R.K. Bioactivity of essential oil from Zingiber officinale (Zingiberaceae) against three stored-product insect species. J. Essent. Oil Bear. Plants 2012, 15, 122-133. [CrossRef]

219. Kheirkhah, M.; Ghasemi, V.; Yazdi, A.K.; Rahban, S. Chemical composition and insecticidal activity of essential oil from Ziziphora clinopodioides Lam. used against the Mediterranean flour moth, Ephestia kuehniella Zeller. J. Plant. Prot. Res. 2015, 55, 260. [CrossRef]

220. McDonald, L.L.; Guy, R.H.; Speirs, R.D. Preliminary Evaluation of New Candidate Materials as Toxicants, Repellents, and Attractants Against Stored-Product Insects; US Department of Agriculture Marketing Research Report 882; US Department of Agriculture: Washington, DC, USA, 1970; p. 8.

221. Chu, S.S.; Feng Hu, J.; Liu, Z.L. Composition of essential oil of Chinese Chenopodium ambrosioides and insecticidal activity against maize weevil, Sitophilus zeamais. Pest Manag. Sci. 2011, 67, 714-718. [CrossRef] [PubMed]

222. Chu, S.S.; Du, S.S.; Liu, Z.L. Fumigant compounds from the essential oil of Chinese Blumea balsamifera leaves against the maize weevil (Sitophilus zeamais). J. Chem. 2013, 2013, 289874. [CrossRef]

223. Bishop, G.W. The Comparative Bionomics of American Cryptolestes (Coleoptera-Cucujidae) that Infest Stored Grain. Ann. Entomol. Soc. Am. 1959, 52, 657-665. [CrossRef]

224. Edde, P.A. A review of the biology and control of Rhyzopertha dominica (F.) the lesser grain borer. J. Stored Prod. Res. 2012, 48, 1-18. [CrossRef]

225. Faroni, L.; García-Mari, F. Influencia de la temperatura sobre los parámetros biológicos de Rhyzopertha dominica (F.). Boletín Sanidad Vegetal Plagas 1992, 18, 455-467.

226. Shojaaddini, M.; Moharramipour, S.; Sahaf, B. Fumigant toxicity of essential oil from Carum copticum against Indian meal moth, Plodia interpunctella. J. Plant. Prot. Res. 2008, 48, 4. [CrossRef]

227. Shin, S.W.; Jeon, J.H.; Yun, C.-S.; Jeong, S.A.; Kim, J.-A.; Park, D.-S.; Shin, Y.; Oh, H.-W. Species-specific interactions between plant metabolites and insect juvenile hormone receptors. J. Chem. Ecol. 2018, 44, 1022-1029. [CrossRef] [PubMed]

228. Moharramipour, S.; Negahban, M. Plant essential oils and pest management. In Basic and Applied Aspects of Biopesticides; Springer: New Delhi, India, 2014; pp. 129-153.

229. Pavela, R.; Benelli, G. Essential Oils as Ecofriendly Biopesticides? Challenges and Constraints. Trends Plant Sci. 2016, 21, 1000-1007. [CrossRef] [PubMed]

230. Menossi, M.; Ollier, R.P.; Casalongué, C.A.; Alvarez, V.A. Essential oil-loaded bio-nanomaterials for sustainable agricultural applications. J. Chem. Technol. Biotechnol. 2021, 96, 2109-2122. [CrossRef]

231. Ogendo, J.O.; Deng, A.L.; Birech, R.J.; Bett, P.K. Plant-Based Products as Control Agents of Stored-Product Insect Pests in the Tropics. In Progress in Food Preservation; Wiley-Blackwell Publishers: London, UK, 2012; pp. 581-601. 